ISSN 2075-1729

www.mdpi.com/journal/life

Review

\title{
Simple Organics and Biomonomers Identified in HCN Polymers: An Overview
}

\author{
Marta Ruiz-Bermejo $^{1, *}$, María-Paz Zorzano ${ }^{2}$ and Susana Osuna-Esteban ${ }^{1}$ \\ 1 Departamento de Evolución Molecular, Centro de Astrobiología (CSIC-INTA), \\ Ctra, Torrejón-Ajalvir, km 4, 28850-Torrejón de Ardoz, Madrid, Spain; \\ E-Mail: osunaes@cab.inta-csic.es \\ 2 Departamento Instrumentación Avanzada, Centro de Astrobiología (CSIC-INTA), \\ Ctra, Torrejón-Ajalvir, km 4, 28850-Torrejón de Ardoz, Madrid, Spain; \\ E-Mail: zorzanomm@cab.inta-csic.es
}

* Author to whom correspondence should be addressed; E-Mail: ruizbm@cab.inta-csic.es; Tel.: +34-915-206-402; Fax: +34-915-506-410.

Received: 15 April 2013; in revised form: 18 June 2013 / Accepted: 28 June 2013 /

Published: 29 July 2013

\begin{abstract}
Hydrogen cyanide $(\mathrm{HCN})$ is a ubiquitous molecule in the Universe. It is a compound that is easily produced in significant yields in prebiotic simulation experiments using a reducing atmosphere. HCN can spontaneously polymerise under a wide set of experimental conditions. It has even been proposed that HCN polymers could be present in objects such as asteroids, moons, planets and, in particular, comets. Moreover, it has been suggested that these polymers could play an important role in the origin of life. In this review, the simple organics and biomonomers that have been detected in HCN polymers, the analytical techniques and procedures that have been used to detect and characterise these molecules and an exhaustive classification of the experimental/environmental conditions that favour the formation of $\mathrm{HCN}$ polymers are summarised. Nucleobases, amino acids, carboxylic acids, cofactor derivatives and other compounds have been identified in $\mathrm{HCN}$ polymers. The great molecular diversity found in HCN polymers encourages their placement at the central core of a plausible protobiological system.
\end{abstract}

Keywords: HCN polymers; prebiotic synthesis; nucleobases; amino acids; carboxylic acids; chromatographic techniques 


\section{Introduction}

Different strategies for the prebiotic synthesis of biomonomers have been designed assuming that the reactive precursors [i.e., hydrogen cyanide $(\mathrm{HCN})$, formaldehyde $\left(\mathrm{CH}_{2} \mathrm{O}\right)$, formamide $\left(\mathrm{HCONH}_{2}\right)$, ammonium cyanide $\left(\mathrm{NH}_{4} \mathrm{CN}\right)$, cyanoacetylene and others] were available in adequate concentrations on the primitive Earth or elsewhere [1]. The prebiotic transformation most likely occurred in water (oceans, lagoons or lakes) at moderate $\mathrm{pH}$ values or in solid-state conditions caused by the slow evaporation of water.

HCN (also known as prussic acid) is considered to be one of the most important and versatile building blocks for the construction of biomolecules. HCN was prepared for the first time by $\mathrm{C}$. W. Scheele (1742-1786). He heated blood with $\mathrm{KOH}$ and charcoal and obtained what he called "Blutlage", which he distilled with sulphuric acid [2]. The oligomerisation of HCN was first observed by J. L. Proust in 1806 [3]. In 1874, R. Wipperman published the results of his studies on the conversion of aqueous $\mathrm{HCN}$ into its trimer (aminomalonic acid dinitrile), which subsequently hydrolysed and decarboxylated to produce glycine, which he separated by crystallisation and identified by elemental analysis [4]. Only one year later, E. Pflüger published one of the earliest chemical speculations concerning the origin of "living proteins" from cyano compounds: "cyanogen and its compounds had plenty of time and opportunity to follow their great tendency to transformation and polymerisation and, by the addition of oxygen and later water and salts, to change to a labile protein, which constitutes living matter" [5]. Almost one century later, J. Oró claimed the first prebiotic synthesis of adenine from refluxed solutions of concentrated ammonium cyanide [6]. Since then, HCN polymerisation has generally been considered the preferential prebiotic route for the synthesis of purines and pyrimidine derivatives. Thus, it has been suggested that HCN polymers may be important substances in the first stages of the chemical evolution of life.

From an astrobiological point of view, $\mathrm{HCN}$ is a very interesting reactant. $\mathrm{HCN}$ molecules and $\mathrm{CN}$ radicals are ubiquitous in the Universe. This molecule is observed in planetary and interstellar locations and is easily produced in plausible prebiotic environments. HCN has been detected in remote galaxies [7], in interstellar clouds [8,9], notably in star-forming regions [10-14], in reflection nebulae [15-18], in planetary nebulae [19], in interplanetary dust [20,21], in circumstellar envelopes and discs [22-27], in comets [28-31], in meteorites [32], in the atmospheres of the outer planets and their moons [33-37] and, in a terrestrial context, in volcanic gases and hydrothermal vents [38,39]. $\mathrm{HCN}$ is the major product when appropriate gas mixtures are subjected to intense physical energy, such as from electric discharges, UV radiation or shock waves [40-44].

$\mathrm{HCN}$ can spontaneously polymerise in the presence of bases, such as ammonia and free radicals from ionising radiation, and the spontaneous polymerisation occurs over a wide range of temperatures and pressures in both polar (water) and non-polar (hydrocarbon) solvents and surfaces. Recently, a synthesis has also described the formation of an HCN polymer from formamide [45]. The HCN polymers, also known as HCN oligomers, azulmic acid or azulmin, are heterogeneous solids ranging in colour from yellow or orange to brown or black, depending on the degrees of polymerisation and cross-linking. The structures of $\mathrm{HCN}$ polymers have not been fully characterised and remain controversial due to their complex and heterogeneous nature. Several models try to explain the complex structure of $\mathrm{HCN}$ polymers [46-52]. However, the structure, nature and behaviour of $\mathrm{HCN}$ 
polymers are intriguing issues, and although some aspects have been resolved, many questions remain unanswered. Active research into the nature and properties of the HCN polymers is still in progress [53-57].

A number of studies were carried out over the last fifty years to define the molecules present in the HCN polymers, and excellent reviews have been published addressing this topic [58-61]. Nevertheless, comparing the products obtained under different reaction conditions or different analytical procedures is difficult. In this review, an updated and comprehensive catalogue of the simple organics and biomonomers found in the HCN polymers is shown. The experimental/environmental conditions for the polymerisation of $\mathrm{HCN}$ are described for each compound as well as the analytical techniques and procedures that should be used to detect and characterise them. Additionally, some mechanistic aspects are described, particularly for the production of nucleobases.

\section{Amino Acids}

In 1953, S. L. Miller demonstrated with his famous and successful experiment that a prebiotic formation of amino acids was possible [62]. After this pioneering work, J. Oró investigated the possible role of the aqueous polymerisation of $\mathrm{HCN}$ in the amino acids production [63]. Glycine, alanine and aspartic acid were identified by paper chromatography after heating a concentrated ammoniacal solution of $\mathrm{HCN}$ at $70{ }^{\circ} \mathrm{C}$ for 25 days. On the basis of this experiment, many other conditions have been used to investigate the $\mathrm{HCN}$ polymerisation processes, which can be grouped into four rough categories: (i) heating experiments; (ii) room temperature experiments; (iii) radiation experiments; (iv) freezing experiments. In the heating experiments, ammoniacal solutions of HCN (2.2-0.1 M) are heated at $100-38{ }^{\circ} \mathrm{C}$ for $1-30$ days. In the room temperature experiments, similar solutions of $\mathrm{HCN}$ are used $(1-0.1 \mathrm{M})$, but the reaction times are increased from 1 month to 18 months. It is well known that only concentrated solutions of $\mathrm{HCN}(>0.01 \mathrm{M})$ ) can polymerise and produce nucleic-acid bases and amino acids, whereas in dilute solutions, hydrolysis becomes dominant [64]. Taking into account the production rates of $\mathrm{HCN}$ in the primitive atmosphere and the experimental hydrolysis rates, the steady state concentration of $\mathrm{HCN}$ in the primitive ocean could be in the range of $4 \times 10^{-6}-2 \times 10^{-8} \mathrm{M}$ at a pH value of 8 between $0{ }^{\circ} \mathrm{C}$ and $25{ }^{\circ} \mathrm{C}$ [65]. Miyakawa et al. [66] estimated this concentration to be approximately $2 \times 10^{-6} \mathrm{M}$ at $\mathrm{pH}=8$ and $0{ }^{\circ} \mathrm{C}$. Therefore, if $\mathrm{HCN}$ polymerisation was actually important for the production of the first and essential biomolecules, there must have been routes by which diluted $\mathrm{HCN}$ solutions were efficiently concentrated. Because $\mathrm{HCN}$ is more volatile than water, it cannot be concentrated by evaporation if the $\mathrm{pH}$ is lower than the $\mathrm{pK}_{\mathrm{a}}$ of $\mathrm{HCN}\left(9.2\right.$ at $\left.25^{\circ} \mathrm{C}\right)$. Therefore, an alternative and plausible mechanism is eutectic freezing. Thus, using freezing conditions, Miller and co-workers achieved the synthesis of glycine, alanine and aspartic acid from a frozen ammonium cyanide solution ( $\mathrm{HCN} 0.1 \mathrm{M}$ plus $\mathrm{NH}_{3} 0.1 \mathrm{M}, \mathrm{pH} 9.2$ ) that had been held at from -20 to $-78{ }^{\circ} \mathrm{C}$ for periods of 2 months to 25 years [67]. The eutectic phase of an aqueous HCN solution, which occurs at $-23.4{ }^{\circ} \mathrm{C}$, contains $74.5 \% \mathrm{HCN}$ (25 M) [64]. In an early terrestrial context, because eutectic formation requires complete freezing, it is possible to propose the shallowest pools or areas under the most severe glacier conditions as more favourable sites for HCN oligomerisation. Additionally, it is possible to suggest other planetary objects in the Solar Systems that have these conditions, such as the icy moons of Jupiter [67]. The characteristics of eutectic systems merit further 
investigation in the contexts of chemical evolution and planetary exploration, in particular, in the context of the next mission to Europa, Ganymede and Callisto (JUICE mission, 2020).

Moreover, ionising radiation can also catalyse the polymerisation of HCN. Among the available sources of energy that may have enabled chemical evolution in the early Earth were UV radiation and radioactivity [68]. Abelson showed the production of glycine, alanine, aspartic acid and serine using diluted solutions of HCN at pH 8-9 and UV radiation [69]. Draganic and co-workers have studied the amino acids formed by the acid hydrolysis of oligomers from ${ }^{60} \mathrm{Co}$-radiolysis of $\mathrm{HCN}$ [70,71].

Table 1 describes the conditions of synthesis and hydrolysis for HCN polymers and the analytical methods for the identification of the amino acids. One can see that most of the experiments were performed in aqueous solution, and only a few experiments were carried out under anhydrous conditions. Using the four groups of conditions described, twenty four amino acids were identified (see Figure 1a). In most cases, acid hydrolysis of the HCN polymers was necessary before their detection and identification. The HCN polymers yield twelve of the twenty protein amino acids (Figure 1b). It is interesting to note that glycine and aspartic acid are the only amino acids that can be identified under all conditions assayed (twenty three sets of experimental conditions). Moreover, glycine is the amino acid that is always obtained with the greatest yield. Labadie et al. estimated a glycine yield of approximately $0.11 \mathrm{~mol} / \mathrm{L}$ [72]. In contrast, glycinamide, aminomalonic acid and 2,3-aminopropionic acid were detected under unique reaction conditions. Sarcosine and 2-methyl aspartic acid have only been detected under $\gamma$-radiation conditions and 4-amino-n-butyric acid and ornithine under hot conditions at $90{ }^{\circ} \mathrm{C}$. Diaminosuccinic acid was only found in room temperature experiments by Ferris and co-worker (Figure 1a).

Taking into consideration that most of the experiments shown in Table 1 were carried out between 1961 and 1984 and great developments in chromatography and analytical techniques have taken place since that time, further identification of the organic compounds in HCN polymers may be necessary. The identification of amino acids seems to depend on the experimental conditions of the polymerisation, the sample preparation and the analytical tools used. The discovery of alkaline lakes or soda lakes with $\mathrm{pH}$ values between 9 and 12 presents a new possibility for the concentration of cyanide in tidal beaches by evaporation and encourages a revision of the experiments at moderate temperatures and relatively high concentration of cyanide. The mechanism for the formation of amino acids from HCN polymerisation has not been described, but it has been suggested that the trimer (aminomalononitrile, AMN) or tetramer (diaminomaleonitrile, DAMN) of HCN might be involved in the synthesis. Studies of the formation of amino acids from AMN and DAMN were conducted by Matthews and co-worker [73,74] as well as Ferris and co-worker [64]. 
Table 1. Amino acids identified in Hydrogen Cyanide ( $\mathrm{HCN})$ polymers. The reaction conditions are described together with the material analysed: soluble fraction (solution or soluble oligomers), insoluble fraction (brown or dark precipitates) or raw reaction. $\mathrm{c}(\mathrm{M})=$ initial molar concentration of the reactant in aqueous solution; $\mathrm{HCN}_{(\mathrm{L})}=\mathrm{HCN}$ in liquid phase; $\mathrm{HCN}_{(\mathrm{G})}=\mathrm{HCN}$ in gas phase; $\mathrm{d}=\mathrm{days} ; \mathrm{m}=\mathrm{moths} ; \mathrm{y}=\mathrm{years}$; Non hydrolysis = no additional hydrolysis (acid, basic or neutral) was made over the final product analysed; Acid $=\mathrm{HCl} 6 \mathrm{~N} / 100-110{ }^{\circ} \mathrm{C} / 16-24 \mathrm{~h}$; Basic $=\mathrm{NaOH} 0.1 \mathrm{~N} / 100{ }^{\circ} \mathrm{C} / 6 \mathrm{~h} ;$ Neutral $=\mathrm{NaOH}$ pH $8-8.5 / 110{ }^{\circ} \mathrm{C} / 6-24 \mathrm{~h} ;$ GC-MS = Gas Chromatography-Mass Spectrometry; $\mathrm{AAA}=$ Automatic amino acid analyzer; $\mathrm{PC}=$ paper chromatography; HPLC $=$ High performance liquid chromatography.

\begin{tabular}{|c|c|c|c|c|c|c|}
\hline Compound & $\begin{array}{c}\text { Starting } \\
\text { material, c (M) }\end{array}$ & $\mathbf{T}\left({ }^{\circ} \mathrm{C}\right) / \mathrm{t} /$ Catalyst & $\begin{array}{c}\text { Final product } \\
\text { analysed }\end{array}$ & Hydrolysis & $\begin{array}{c}\text { Method of } \\
\text { identification }\end{array}$ & Reference \\
\hline \multirow[t]{23}{*}{ Glycine } & $\mathrm{HCN}, 0.2$ & $100 / 1 \mathrm{~d} /-$ & Raw mixture & Acid & GC-MS & [75] \\
\hline & $\mathrm{NH}_{4} \mathrm{CN}, 1$ & $90 / 4 \mathrm{~h} /-$ & Brown precipitate & Acid & AAA & [76] \\
\hline & $\mathrm{HCN}, 1$ & $90 / 4 \mathrm{~h} / \mathrm{NH}_{4} \mathrm{OH}(\mathrm{pH} 9.58)$ & Black solid & Acid & 2D-PC & [72] \\
\hline & $\mathrm{HCN}, 1.5$ & $90 / 18 \mathrm{~h} / \mathrm{NH}_{3}$ & Soluble oligomers & Acid & 2D-PC, AAA & [77] \\
\hline & $\mathrm{HCN}, 1.5$ & $70 / 5 \mathrm{~d} / \mathrm{NH}_{4} \mathrm{OH}$ & Soluble oligomers & Non hydrolysis & $\mathrm{PC}$ & [78] \\
\hline & $\mathrm{HCN}, 2.2$ & $70 / 25 \mathrm{~d} / \mathrm{NH}_{4} \mathrm{OH}$ & Soluble oligomers & Non hydrolysis & $\mathrm{PC}$ & [63] \\
\hline & $\mathrm{NaCN}, 1$ & $38 / 3-30 \mathrm{~d} / \mathrm{NH}_{4} \mathrm{Cl}$ & Black solid & Acid & GC-MS & {$[46]$} \\
\hline & $\mathrm{HCN}_{(\mathrm{L})}$ & r.t. $/ 4$ w/anhydrous $\mathrm{NH}_{3}$ & Dark solid residue & Acid & AAA & [51] \\
\hline & $\mathrm{NaCN}, 1$ & r.t./3 m/pH $9.2(\mathrm{HCl})$ & Soluble oligomers & Acid, basic and neutral & GC-MS & {$[79]$} \\
\hline & $\mathrm{HCN}, 0.1$ & r.t. $/ 1-6 \mathrm{~m} / \mathrm{NH}_{4} \mathrm{OH}(\mathrm{pH} 9.2)$ & Soluble oligomers & Acid & AAA, GC-MS & {$[80,81]$} \\
\hline & $\mathrm{HCN}, 0.1$ & r.t. $/ 4-12 \mathrm{~m} / \mathrm{NH}_{4} \mathrm{OH}(\mathrm{pH} 9.2)$ & Soluble oligomers & Acid and neutral & GC-MS & [82] \\
\hline & $\mathrm{HCN}, 0.1$ & r.t./18 m/ $\mathrm{NH}_{4} \mathrm{OH}(\mathrm{pH} 9.2)$ & Soluble oligomers & Acid, Basic and neutral & GC-MS & [79] \\
\hline & $\mathrm{HCN}, 0.002-0.1$ & r.t./UV radiation (Hg lamp)/pH (8-9) & Soluble oligomers & Acid & AAA & [69] \\
\hline & $\mathrm{HCN}_{(\mathrm{G})}$ & r.t./UV radiation (Hg lamp) & Solid & Acid & AAA & [83] \\
\hline & $\mathrm{HCN}, 0.004-0.1$ & r.t. $/ \gamma$-radiation $\left({ }^{60} \mathrm{Co}\right.$ source $)$ & Raw mixture & Acid & PC, AAA, GC-MS & [84] \\
\hline & $\mathrm{HCN}, 0.1$ & r.t. $/ \gamma$-radiation $\left({ }^{60} \mathrm{Co}\right.$ source $) / \mathrm{pH} 6$ & Raw mixture & Acid & AAA, GC-MS & [70] \\
\hline & $\mathrm{HCN}, 0.1$ & r.t. $/ \gamma$-radiation $\left({ }^{60} \mathrm{Co}\right.$ source $) / \mathrm{NH}_{3} / \mathrm{pH} 9$ & Solution & Acid & AAA, GC-MS & [70] \\
\hline & $\mathrm{NaCN}, 0.1$ & r.t. $/ \gamma$-radiation $\left({ }^{60} \mathrm{Co}\right.$ source $) / \mathrm{pH} 11.3$ & Solution & Acid & AAA, GC-MS & [70] \\
\hline & $\mathrm{HCN}, 0.1$ & r.t. $-40{ }^{\circ} \mathrm{C} / \gamma$-radiation $\left({ }^{60} \mathrm{Co}\right.$ source $) / \mathrm{NH}_{3} / \mathrm{pH} 9$ & Solution & Acid & AAA, GC-MS & [71] \\
\hline & $\mathrm{HCN}, 1.5$ & Refrigerator $/ 4 \mathrm{~d} / \mathrm{NH}_{3}$ & Black Solid & Acid & AAA & [51] \\
\hline & $\mathrm{HCN}, 0.1$ & $-20 / 2 \mathrm{~m} / \mathrm{NH}_{3} / \mathrm{pH} 9.2$ & Solution & Acid & HPLC & [67] \\
\hline & $\mathrm{HCN}, 0.1$ & $-20 / 25 \mathrm{y} / \mathrm{NH}_{3} / \mathrm{pH} 9.2$ & Solution & Acid & HPLC & [67] \\
\hline & $\mathrm{HCN}, 0.1$ & $-78 / 25 \mathrm{y} / \mathrm{NH}_{3} / \mathrm{pH} 9.2$ & Solution & Acid & HPLC & [67] \\
\hline
\end{tabular}


Table 1. Cont.

\begin{tabular}{|c|c|c|c|c|c|c|}
\hline Compound & $\begin{array}{c}\text { Starting } \\
\text { material, c (M) }\end{array}$ & $\mathrm{T}\left({ }^{\circ} \mathrm{C}\right) / \mathrm{t} /$ Catalyst & $\begin{array}{c}\text { Final product } \\
\text { analysed }\end{array}$ & Hydrolysis & $\begin{array}{c}\text { Method of } \\
\text { identification }\end{array}$ & Reference \\
\hline Glycinamide & $\mathrm{HCN}, 1.5$ & $70 / 5 \mathrm{~d} / \mathrm{NH}_{4} \mathrm{OH}$ & Soluble oligomers & Non hydrolysis & $\mathrm{PC}$ & [78] \\
\hline $\begin{array}{l}\text { Aminomalonic } \\
\text { acid }\end{array}$ & $\mathrm{NaCN}, 1$ & $38 / 3-30 \mathrm{~d} / \mathrm{NH}_{4} \mathrm{Cl}$ & Black solid & Acid & GC-MS & [46] \\
\hline \multirow[t]{22}{*}{ Alanine } & $\mathrm{HCN}, 1.5$ & $100 / 1 \mathrm{~d} /-$ & Raw mixture & Acid & GC-MS & [75] \\
\hline & $\mathrm{NH} 4 \mathrm{CN}, 1$ & $90 / 4 \mathrm{~h} /-$ & Brown precipitate & Acid & AAA & [76] \\
\hline & $\mathrm{HCN}, 1$ & $90 / 4 \mathrm{~h} / \mathrm{NH}_{4} \mathrm{OH}(\mathrm{pH} 9.58)$ & Black solid & Acid & 2D-PC & [72] \\
\hline & $\mathrm{HCN}, 1.5$ & $90 / 18 \mathrm{~h} / \mathrm{NH}_{3}$ & Soluble oligomers & Acid & 2D-PC, AAA & [77] \\
\hline & $\mathrm{HCN}, 1.5$ & $70 / 5 \mathrm{~d} / \mathrm{NH}_{4} \mathrm{OH}$ & Soluble oligomers & Non hydrolysis- & PC & [78] \\
\hline & $\mathrm{HCN}, 2.2$ & $70 / 25 \mathrm{~d} / \mathrm{NH}_{4} \mathrm{OH}$ & Soluble oligomers & Non hydrolysis & $\mathrm{PC}$ & {$[63]$} \\
\hline & $\mathrm{HCN}_{(\mathrm{L})}$ & r.t. $/ 4$ w/anhydrous $\mathrm{NH}_{3}$ & Dark solid residue & Acid & AAA & {$[51]$} \\
\hline & $\mathrm{NaCN}, 1$ & r.t./3 m/pH $9.2(\mathrm{HCl})$ & Soluble oligomers & Acid, basic and neutral & GC-MS & [79] \\
\hline & $\mathrm{HCN}, 0.1$ & r.t./1-6 m/NH $\mathrm{NH}_{4} \mathrm{OH}(\mathrm{pH} 9.2)$ & Soluble oligomers & Acid & AAA, GC-MS & {$[80,81]$} \\
\hline & $\mathrm{HCN}, 0.1$ & r.t. $/ 4-12 \mathrm{~m} / \mathrm{NH}_{4} \mathrm{OH}(\mathrm{pH} 9.2)$ & Soluble oligomers & Acid and neutral & GC-MS & {$[82]$} \\
\hline & $\mathrm{HCN}, 0.1$ & r.t./18 m/ $/ \mathrm{NH}_{4} \mathrm{OH}(\mathrm{pH} 9.2)$ & Soluble oligomers & Acid, Basic and neutral & GC-MS & [79] \\
\hline & $\mathrm{HCN}, 0.002-0.1$ & r.t./UV radiation (Hg lamp)/pH (8-9) & Solution & Acid & AAA & [69] \\
\hline & $\mathrm{HCN}_{(\mathrm{G})}$ & r.t./UV radiation (Hg lamp) & Solid & Acid & AAA & [83] \\
\hline & $\mathrm{HCN}, 0.004-0.1$ & r.t. $/ \gamma$-radiation $\left({ }^{60} \mathrm{Co}\right.$ source $)$ & Raw mixture & Acid & PC, AAA, GC-MS & [84] \\
\hline & HCN, 0.1 & r.t. $/ \gamma$-radiation $\left({ }^{60} \mathrm{Co}\right.$ source $) / \mathrm{pH} 6$ & Raw mixture & Acid & AAA, GC-MS & [70] \\
\hline & $\mathrm{HCN}, 0.1$ & r.t. $/ \gamma$-radiation $\left({ }^{60} \mathrm{Co}\right.$ source $) / \mathrm{NH}_{3} / \mathrm{pH} 9$ & Solution & Acid & AAA, GC-MS & [70] \\
\hline & $\mathrm{NaCN}, 0.1$ & r.t. $/ \gamma$-radiation $\left({ }^{60} \mathrm{Co}\right.$ source $) / \mathrm{pH} 11.3$ & Solution & Acid & AAA, GC-MS & {$[70]$} \\
\hline & $\mathrm{HCN}, 0.1$ & r.t. $-40{ }^{\circ} \mathrm{C} / \gamma$-radiation $\left({ }^{60} \mathrm{Co}\right.$ source $) / \mathrm{NH}_{3} / \mathrm{pH} 9$ & Solution & Acid & AAA, GC-MS & [71] \\
\hline & $\mathrm{HCN}, 1.5$ & Refrigerator/ $4 \mathrm{~d} / \mathrm{NH}_{3}$ & Black Solid & Acid & AAA & {$[51]$} \\
\hline & $\mathrm{HCN}, 0.1$ & $-20 / 2 \mathrm{~m} / \mathrm{NH}_{3} / \mathrm{pH} 9.2$ & Solution & Acid & HPLC & {$[67]$} \\
\hline & $\mathrm{HCN}, 0.1$ & $-20 / 25 \mathrm{y} / \mathrm{NH}_{3} / \mathrm{pH} 9.2$ & Solution & Acid & HPLC & {$[67]$} \\
\hline & $\mathrm{HCN}, 0.1$ & $-78 / 25 \mathrm{y} / \mathrm{NH}_{3} / \mathrm{pH} 9.2$ & Solution & Acid & HPLC & {$[67]$} \\
\hline \multirow[t]{6}{*}{$\beta$-alanine } & $\mathrm{HCN}, 1.5$ & $90 / 18 \mathrm{~h} / \mathrm{NH}_{3}$ & Soluble oligomers & Acid & 2D-PC, AAA & [77] \\
\hline & $\mathrm{HCN}, 0.1$ & r.t. $/ 4-12 \mathrm{~m} / \mathrm{NH}_{4} \mathrm{OH}(\mathrm{pH} 9.2)$ & Soluble oligomers & Acid and neutral & GC-MS & {$[82]$} \\
\hline & $\mathrm{HCN}, 0.1$ & r.t. $/ \gamma$-radiation $\left({ }^{60} \mathrm{Co}\right.$ source $) / \mathrm{pH} 6$ & Raw mixture & Acid & AAA, GC-MS & [70] \\
\hline & $\mathrm{HCN}, 0.1$ & r.t. $/ \gamma$-radiation $\left({ }^{60} \mathrm{Co}\right.$ source $) / \mathrm{NH}_{3} / \mathrm{pH} 9$ & Solution & Acid & AAA, GC-MS & [70] \\
\hline & $\mathrm{NaCN}, 0.1$ & r.t. $/ \gamma$-radiation $\left({ }^{60} \mathrm{Co}\right.$ source $) / \mathrm{pH} 11.3$ & Solution & Acid & AAA, GC-MS & [70] \\
\hline & $\mathrm{HCN}, 0.1$ & r.t. $-40{ }^{\circ} \mathrm{C} / \gamma$-radiation $\left({ }^{60} \mathrm{Co}\right.$ source $) / \mathrm{NH}_{3} / \mathrm{pH} 9$ & Solution & Acid & AAA, GC-MS & [71] \\
\hline
\end{tabular}


Table 1. Cont.

\begin{tabular}{|c|c|c|c|c|c|c|}
\hline Compound & $\begin{array}{c}\text { Starting } \\
\text { material, c (M) }\end{array}$ & $\mathrm{T}\left({ }^{\circ} \mathrm{C}\right) / \mathrm{t} /$ Catalyst & $\begin{array}{c}\text { Final product } \\
\text { analysed }\end{array}$ & Hydrolysis & $\begin{array}{c}\text { Method of } \\
\text { identification }\end{array}$ & Reference \\
\hline \multirow[t]{4}{*}{ Sarcosine } & HCN, 0.1 & r.t. $/ \gamma$-radiation $\left({ }^{60} \mathrm{Co}\right.$ source $) / \mathrm{pH} 6$ & Raw mixture & Acid & AAA, GC-MS & [70] \\
\hline & $\mathrm{HCN}, 0.1$ & r.t. $/ \gamma$-radiation $\left({ }^{60} \mathrm{Co}\right.$ source $) / \mathrm{NH}_{3} / \mathrm{pH} 9$ & Solution & Acid & AAA, GC-MS & [70] \\
\hline & $\mathrm{NaCN}, 0.1$ & r.t. $/ \gamma$-radiation $\left({ }^{60} \mathrm{Co}\right.$ source $) / \mathrm{pH} 11.3$ & Solution & Acid & AAA, GC-MS & [70] \\
\hline & $\mathrm{HCN}, 0.1$ & r.t. $-40{ }^{\circ} \mathrm{C} / \gamma$-radiation $\left({ }^{60} \mathrm{Co}\right.$ source $) / \mathrm{NH}_{3} / \mathrm{pH} 9$ & Solution & Acid & AAA, GC-MS & [71] \\
\hline \multirow[t]{13}{*}{ Serine } & $\mathrm{NH} 4 \mathrm{CN}, 1$ & $90 / 4 \mathrm{~h} /-$ & Brown precipitate & Acid & AAA & [76] \\
\hline & $\mathrm{HCN}, 1$ & $90 / 4 \mathrm{~h} / \mathrm{NH}_{4} \mathrm{OH}(\mathrm{pH} 9.58)$ & Black solid & Acid & 2D-PC & [72] \\
\hline & $\mathrm{HCN}, 1.5$ & $90 / 18 \mathrm{~h} / \mathrm{NH}_{3}$ & Soluble oligomers & Acid & 2D-PC, AAA & [77] \\
\hline & $\mathrm{HCN}_{(\mathrm{L})}$ & r.t./.4 w/anhydrous $\mathrm{NH}_{3}$ & Dark solid residue & Acid & AAA & {$[51]$} \\
\hline & $\mathrm{HCN}, 0.1$ & r.t. $/ 1-6 \mathrm{~m} / \mathrm{NH}_{4} \mathrm{OH}(\mathrm{pH} 9.2)$ & Soluble oligomers & Acid & AAA & {$[80,81]$} \\
\hline & $\mathrm{HCN}, 0.002-0.1$ & r.t./UV radiation (Hg lamp)/pH (8-9) & Solution & Acid & AAA & [69] \\
\hline & $\mathrm{HCN}_{(\mathrm{G})}$ & r.t./UV radiation (Hg lamp) & Solid & Acid & AAA & [83] \\
\hline & $\mathrm{HCN}, 0.004$ & r.t. $/ \gamma$-radiation $\left({ }^{60} \mathrm{Co}\right.$ source $)$ & Raw mixture & Acid & PC, AAA, GC-MS & [84] \\
\hline & $\mathrm{HCN}, 0.1$ & r.t. $/ \gamma$-radiation $\left({ }^{60} \mathrm{Co}\right.$ source $) / \mathrm{pH} 6$ & Raw mixture & Acid & AAA & [70] \\
\hline & $\mathrm{HCN}, 0.1$ & r.t. $/ \gamma$-radiation $\left({ }^{60} \mathrm{Co}\right.$ source $) / \mathrm{NH}_{3} / \mathrm{pH} 9$ & Solution & Acid & AAA & [70] \\
\hline & $\mathrm{NaCN}, 0.1$ & r.t. $/ \gamma$-radiation $\left({ }^{60} \mathrm{Co}\right.$ source $) / \mathrm{pH} 11.3$ & Solution & Acid & AAA & [70] \\
\hline & $\mathrm{HCN}, 0.1$ & r.t. $-40{ }^{\circ} \mathrm{C} / \gamma$-radiation $\left({ }^{60} \mathrm{Co}\right.$ source $) / \mathrm{NH}_{3} / \mathrm{pH} 9$ & Solution & Acid & AAA, GC-MS & [71] \\
\hline & $\mathrm{HCN}, 1.5$ & Refrigerator $/ 4 \mathrm{~d} / \mathrm{NH}_{3}$ & Black Solid & Acid & AAA & [51] \\
\hline 2,3-Aminopropioinic acid & $\mathrm{HCN}, 1.5$ & $90 / 18 \mathrm{~h} / \mathrm{NH}_{3}$ & Soluble oligomers & Acid & 2D-PC, AAA & [77] \\
\hline \multirow[t]{14}{*}{ Aspartic acid } & $\mathrm{HCN}, 0.2$ & $100 / 1 \mathrm{~d} /-$ & Raw mixture & Acid & GC-MS & {$[75]$} \\
\hline & $\mathrm{NH}_{4} \mathrm{CN}, 1$ & $90 / 4 \mathrm{~h} /-$ & Brown precipitate & Acid & AAA & {$[76]$} \\
\hline & $\mathrm{HCN}, 1$ & $90 / 4 \mathrm{~h} / \mathrm{NH}_{4} \mathrm{OH}(\mathrm{pH} 9.58)$ & Black solid & Acid & 2D-PC & [72] \\
\hline & $\mathrm{HCN}, 1.5$ & $90 / 18 \mathrm{~h} / \mathrm{NH}_{3}$ & Soluble oligomers & Acid & 2D-PC, AAA & [77] \\
\hline & $\mathrm{HCN}, 1.5$ & $70 / 5 \mathrm{~d} / \mathrm{NH}_{4} \mathrm{OH}$ & Soluble oligomers & Non hydrolysis & $\mathrm{PC}$ & [78] \\
\hline & $\mathrm{HCN}, 2.2$ & $70 / 25 \mathrm{~d} / \mathrm{NH}_{4} \mathrm{OH}$ & Soluble oligomers & Non hydrolysis & $\mathrm{PC}$ & [63] \\
\hline & $\mathrm{NaCN}, 1$ & $38 / 3-30 \mathrm{~d} / \mathrm{NH}_{4} \mathrm{Cl}$ & Black solid & Acid & GC-MS & {$[46]$} \\
\hline & $\mathrm{HCN}_{(\mathrm{L})}$ & r.t. $/ 4$ w/anhydrous $\mathrm{NH}_{3}$ & Dark solid residue & Acid & AAA & [51] \\
\hline & $\mathrm{NaCN}, 1$ & r.t./3 m/pH $9.2(\mathrm{HCl})$ & Soluble oligomers & Acid, basic and neutral & GC-MS & [79] \\
\hline & $\mathrm{HCN}, 0.1$ & r.t./1-6 m/NH $\mathrm{NH}_{4} \mathrm{OH}(\mathrm{pH} 9.2$ ) & Soluble oligomers & Acid & AAA, GC-MS & {$[80,81]$} \\
\hline & $\mathrm{HCN}, 0.1$ & r.t. $/ 4-12 \mathrm{~m} / \mathrm{NH}_{4} \mathrm{OH}(\mathrm{pH} 9.2)$ & Soluble oligomers & Acid and neutral & GC-MS & [82] \\
\hline & $\mathrm{HCN}, 0.1$ & r.t./18 m/NH $\mathrm{NH}_{4} \mathrm{OH}(\mathrm{pH} 9.2)$ & Soluble oligomers & Acid, Basic and neutral & GC-MS & [79] \\
\hline & $\mathrm{HCN}, 0.002-0.1$ & r.t./UV radiation (Hg lamp)/pH (8-9) & Solution & Acid & AAA & [69] \\
\hline & $\mathrm{HCN}_{(\mathrm{G})}$ & r.t./UV radiation (Hg lamp) & Solid & Acid & AAA & [83] \\
\hline
\end{tabular}


Table 1. Cont.

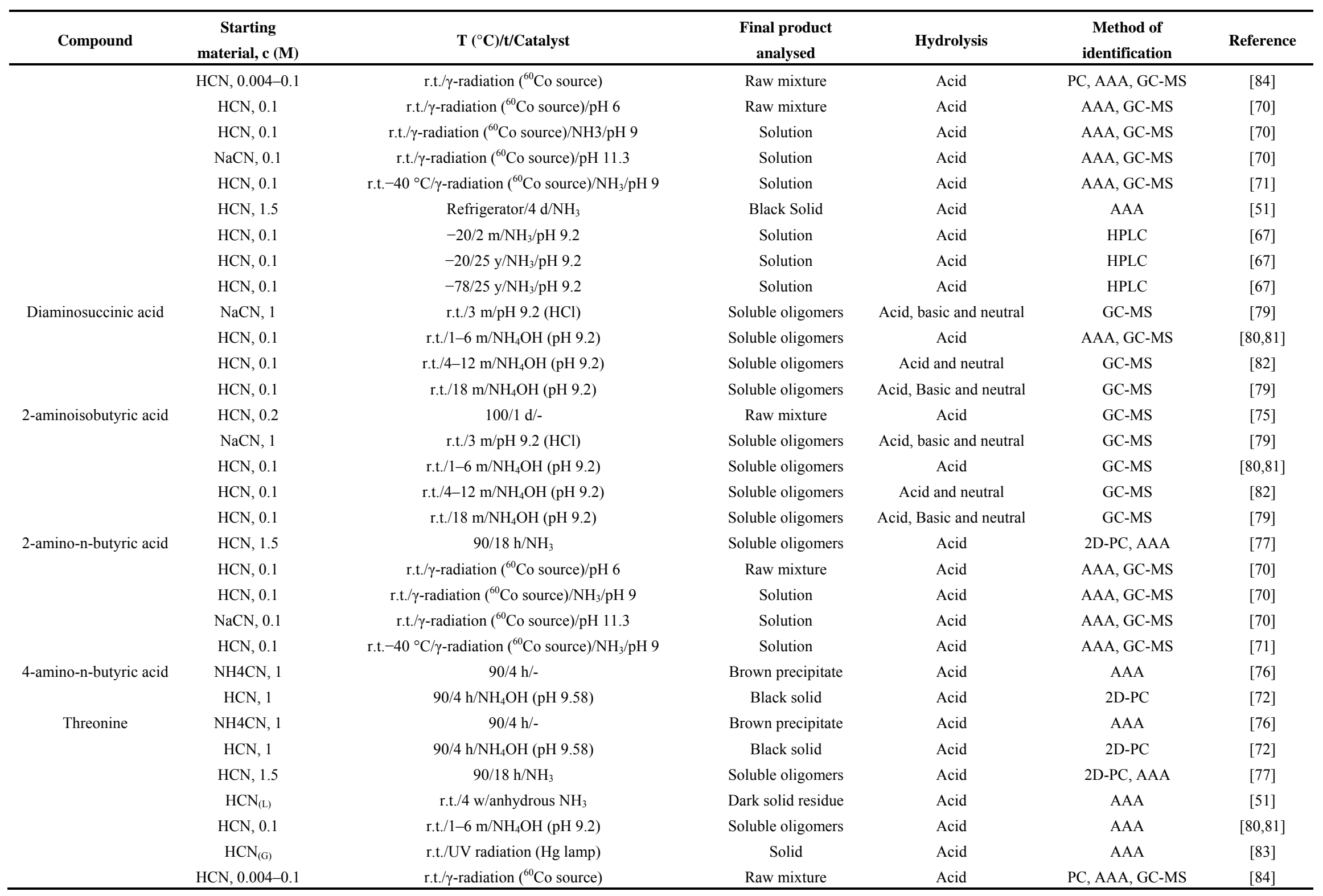


Table 1. Cont.

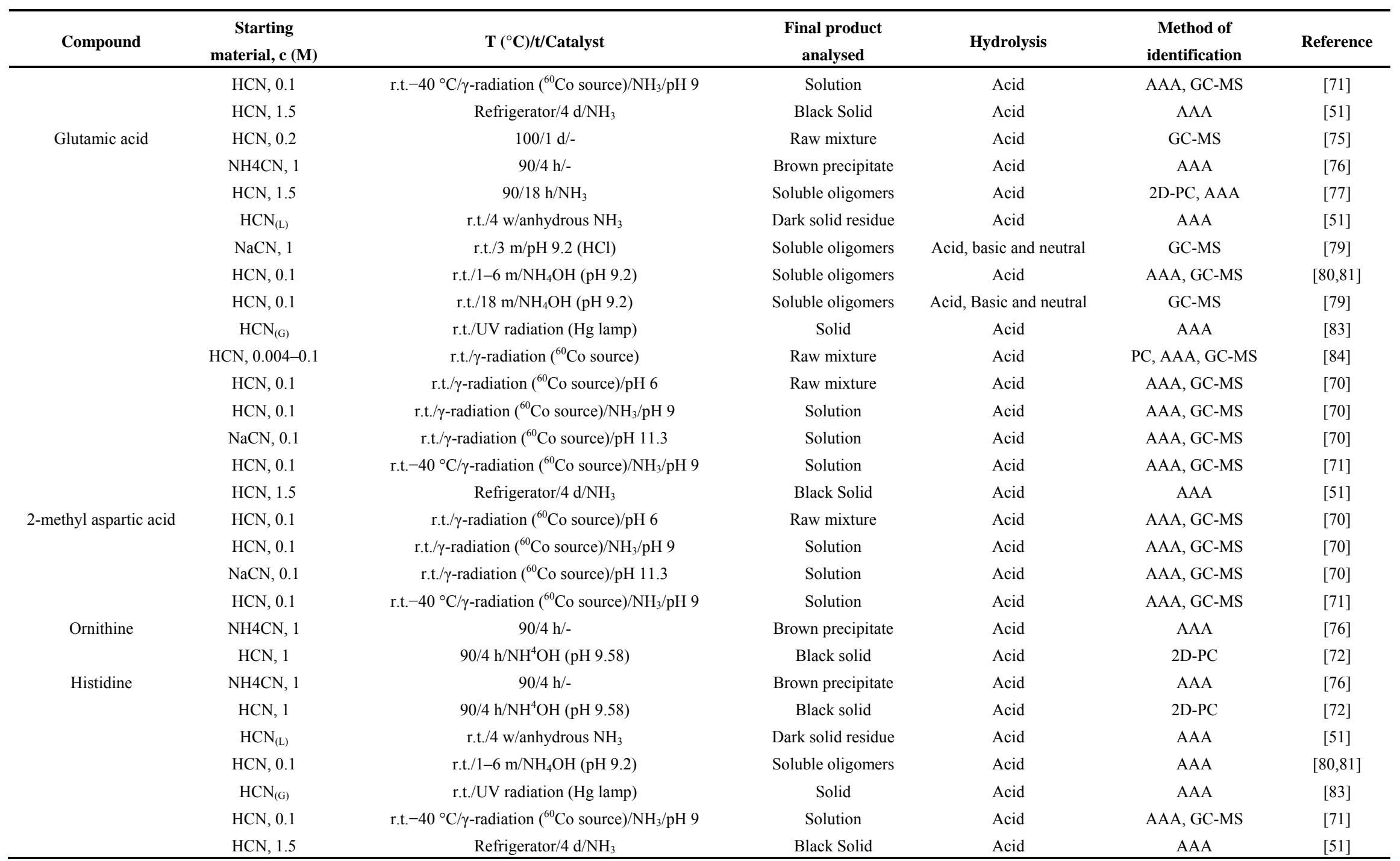


Table 1. Cont.

\begin{tabular}{|c|c|c|c|c|c|c|}
\hline Compound & $\begin{array}{c}\text { Starting } \\
\text { material, c (M) }\end{array}$ & $\mathbf{T}\left({ }^{\circ} \mathrm{C}\right) / \mathrm{t} /$ Catalyst & $\begin{array}{c}\text { Final product } \\
\text { analysed }\end{array}$ & Hydrolysis & $\begin{array}{c}\text { Method of } \\
\text { identification }\end{array}$ & Reference \\
\hline \multirow[t]{5}{*}{ Valine } & $\mathrm{HCN}_{(\mathrm{L})}$ & r.t./4 w/anhydrous $\mathrm{NH}_{3}$ & Dark solid residue & Acid & AAA & {$[51]$} \\
\hline & $\mathrm{HCN}, 0.1$ & r.t./1-6 m/ $\mathrm{NH}_{4} \mathrm{OH}(\mathrm{pH} 9.2)$ & Soluble oligomers & Acid & AAA & {$[80,81]$} \\
\hline & $\mathrm{HCN}_{(\mathrm{G})}$ & r.t./UV radiation (Hg lamp) & Solid & Acid & AAA & {$[83]$} \\
\hline & $\mathrm{HCN}, 0.1$ & r.t. $/ \gamma$-radiation $\left({ }^{60} \mathrm{Co}\right.$ source $)$ & Raw mixture & Acid & PC, AAA, GC-MS & [84] \\
\hline & $\mathrm{HCN}, 1.5$ & Refrigerator $/ 4 \mathrm{~d} / \mathrm{NH}_{3}$ & Black Solid & Acid & AAA & {$[51]$} \\
\hline \multirow[t]{7}{*}{ Isoleucine } & $\mathrm{HCN}, 1.5$ & $90 / 18 \mathrm{~h} / \mathrm{NH}_{3}$ & Soluble oligomers & Acid & 2D-PC, AAA & [77] \\
\hline & $\mathrm{HCN}(\mathrm{L})$ & r.t. $/ 4$ w/anhydrous $\mathrm{NH}_{3}$ & Dark solid residue & Acid & AAA & {$[51]$} \\
\hline & $\mathrm{NaCN}, 1$ & r.t./3 m/pH $9.2(\mathrm{HCl})$ & Soluble oligomers & Acid, basic and neutral & GC-MS & [79] \\
\hline & $\mathrm{HCN}, 0.1$ & r.t. $/ 1-6 \mathrm{~m} / \mathrm{NH}_{4} \mathrm{OH}(\mathrm{pH} 9.2)$ & Soluble oligomers & Acid & AAA, GC-MS & {$[80,81]$} \\
\hline & $\mathrm{HCN}, 0.1$ & r.t./18 m/NH $\mathrm{NH}_{4} \mathrm{OH}(\mathrm{pH} 9.2)$ & Soluble oligomers & Acid, Basic and neutral & GC-MS & {$[79]$} \\
\hline & $\mathrm{HCN}_{(\mathrm{G})}$ & r.t./UV radiation (Hg lamp) & Solid & Acid & AAA & [83] \\
\hline & $\mathrm{HCN}, 1.5$ & Refrigerator $/ 4 \mathrm{~d} / \mathrm{NH}_{3}$ & Black Solid & Acid & AAA & [51] \\
\hline \multirow[t]{5}{*}{ Leucine } & $\mathrm{HCN}, 1.5$ & $90 / 18 \mathrm{~h} / \mathrm{NH}_{3}$ & Soluble oligomers & Acid & 2D-PC, AAA & [77] \\
\hline & $\mathrm{HCN}(\mathrm{L})$ & r.t./4 w/anhydrous $\mathrm{NH}_{3}$ & Dark solid residue & Acid & AAA & [51] \\
\hline & $\mathrm{HCN}, 0.1$ & r.t./1-6 m/ $/ \mathrm{NH}_{4} \mathrm{OH}(\mathrm{pH} 9.2)$ & Soluble oligomers & Acid & AAA & {$[80,81]$} \\
\hline & $\mathrm{HCN}_{(\mathrm{G})}$ & r.t./UV radiation (Hg lamp) & Solid & Acid & AAA & {$[83]$} \\
\hline & $\mathrm{HCN}, 1.5$ & Refrigerator $/ 4 \mathrm{~d} / \mathrm{NH}_{3}$ & Black Solid & Acid & AAA & {$[51]$} \\
\hline \multirow[t]{2}{*}{ Citrulline } & $\mathrm{HCN}, 0.1$ & r.t./1-6 m/NH $\mathrm{NH}_{4} \mathrm{OH}(\mathrm{pH} 9.2)$ & Soluble oligomers & Acid & AAA & {$[80,81]$} \\
\hline & $\mathrm{HCN}, 0.1$ & r.t. $-40{ }^{\circ} \mathrm{C} / \gamma$-radiation $\left({ }^{60} \mathrm{Co}\right.$ source $) / \mathrm{NH}_{3} / \mathrm{pH} 9$ & Solution & Acid & AAA, GC-MS & [71] \\
\hline \multirow[t]{7}{*}{ Lysine } & $\mathrm{NH} 4 \mathrm{CN}, 1$ & $90 / 4 \mathrm{~h} /-$ & Brown precipitate & Acid & AAA & [76] \\
\hline & $\mathrm{HCN}, 1$ & $90 / 4 \mathrm{~h} / \mathrm{NH}_{4} \mathrm{OH}(\mathrm{pH} 9.58)$ & Black solid & Acid & 2D-PC & {$[72]$} \\
\hline & $\mathrm{HCN}_{(\mathrm{L})}$ & r.t./4 w/anhydrous $\mathrm{NH}_{3}$ & Dark solid residue & Acid & AAA & {$[51]$} \\
\hline & $\mathrm{HCN}, 0.1$ & r.t. $/ 1-6 \mathrm{~m} / \mathrm{NH}_{4} \mathrm{OH}(\mathrm{pH} 9.2)$ & Soluble oligomers & Acid & AAA & {$[80,81]$} \\
\hline & $\mathrm{HCN}_{(\mathrm{G})}$ & r.t./UV radiation (Hg lamp) & Solid & Acid & AAA & [83] \\
\hline & $\mathrm{HCN}, 0.1$ & r.t. $-40{ }^{\circ} \mathrm{C} / \gamma$-radiation $\left({ }^{60} \mathrm{Co}\right.$ source $) / \mathrm{NH}_{3} / \mathrm{pH} 9$ & Solution & Acid & AAA, GC-MS & [71] \\
\hline & $\mathrm{HCN}, 1.5$ & Refrigerator $/ 4 \mathrm{~d} / \mathrm{NH}_{3}$ & Black Solid & Acid & AAA & [51] \\
\hline \multirow[t]{2}{*}{ Arginine } & $\mathrm{HCN}_{(\mathrm{L})}$ & r.t./4 w/anhydrous $\mathrm{NH}_{3}$ & Dark solid residue & Acid & AAA & [51] \\
\hline & $\mathrm{HCN}, 1.5$ & Refrigerator/4 d/ $\mathrm{NH}_{3}$ & Black Solid & Acid & AAA & [51] \\
\hline
\end{tabular}


Figure 1. (a) Amino acids detected in Hydrogen Cyanide (HCN) polymers; (b) The protein amino acids found in HCN polymers are shown in colour.

a)

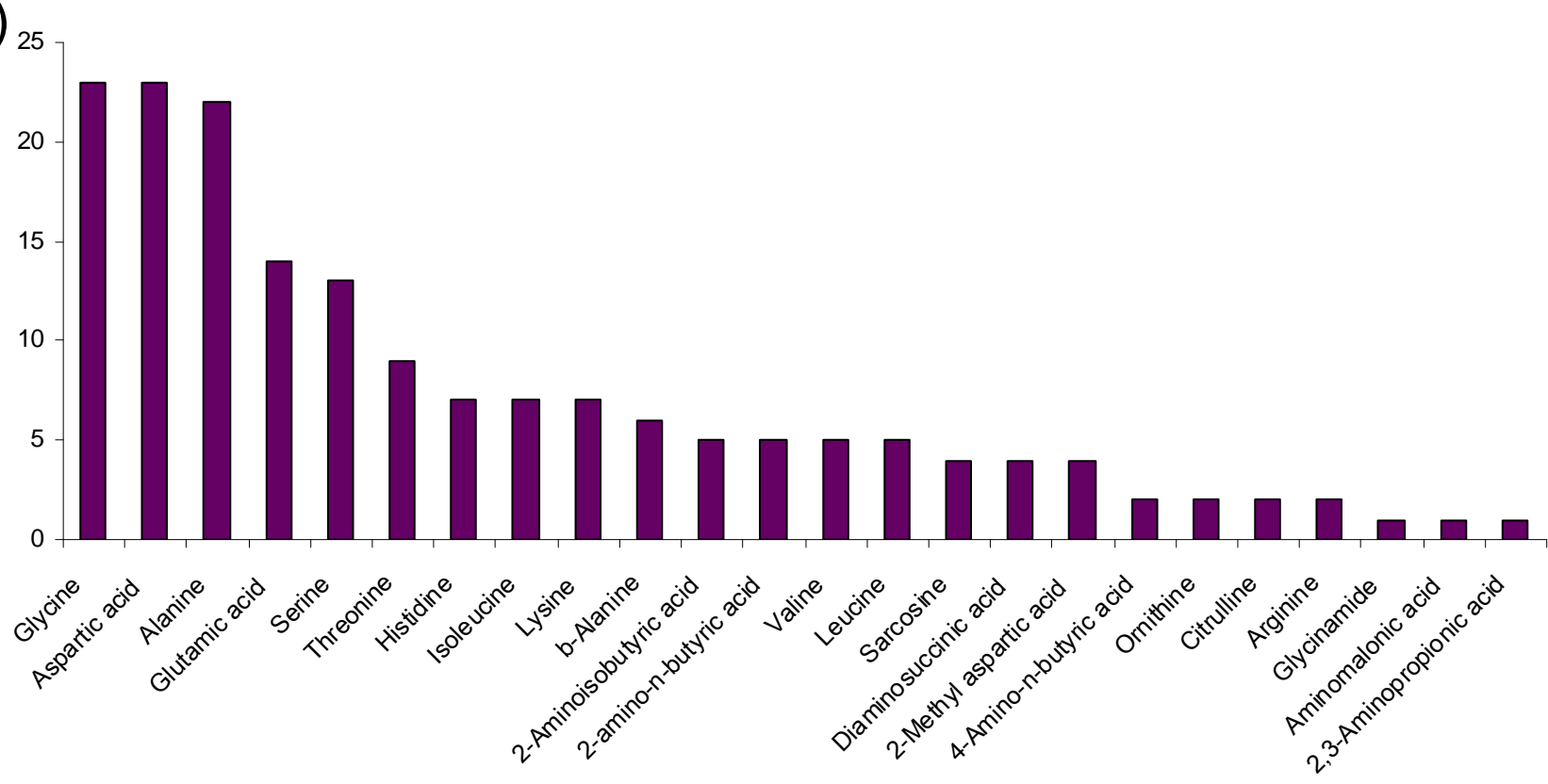

b)
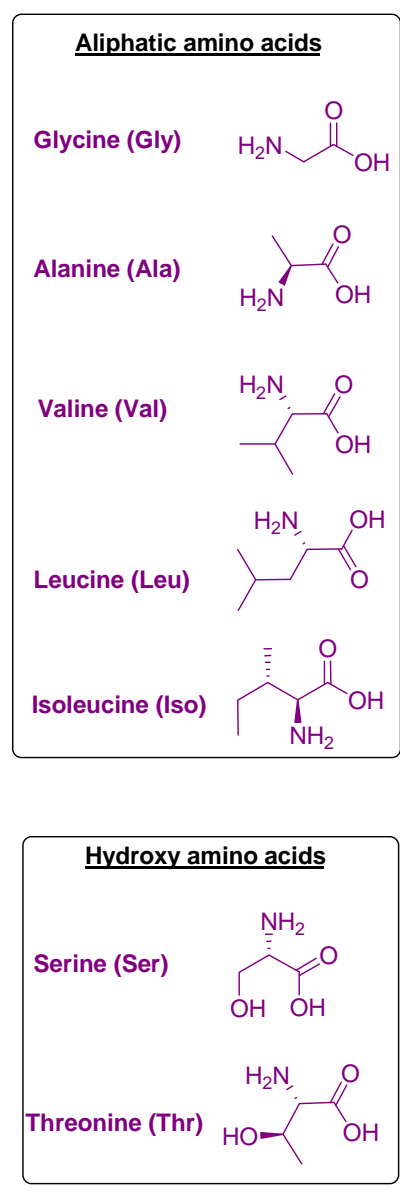

PROTEIN AMINO ACIDS
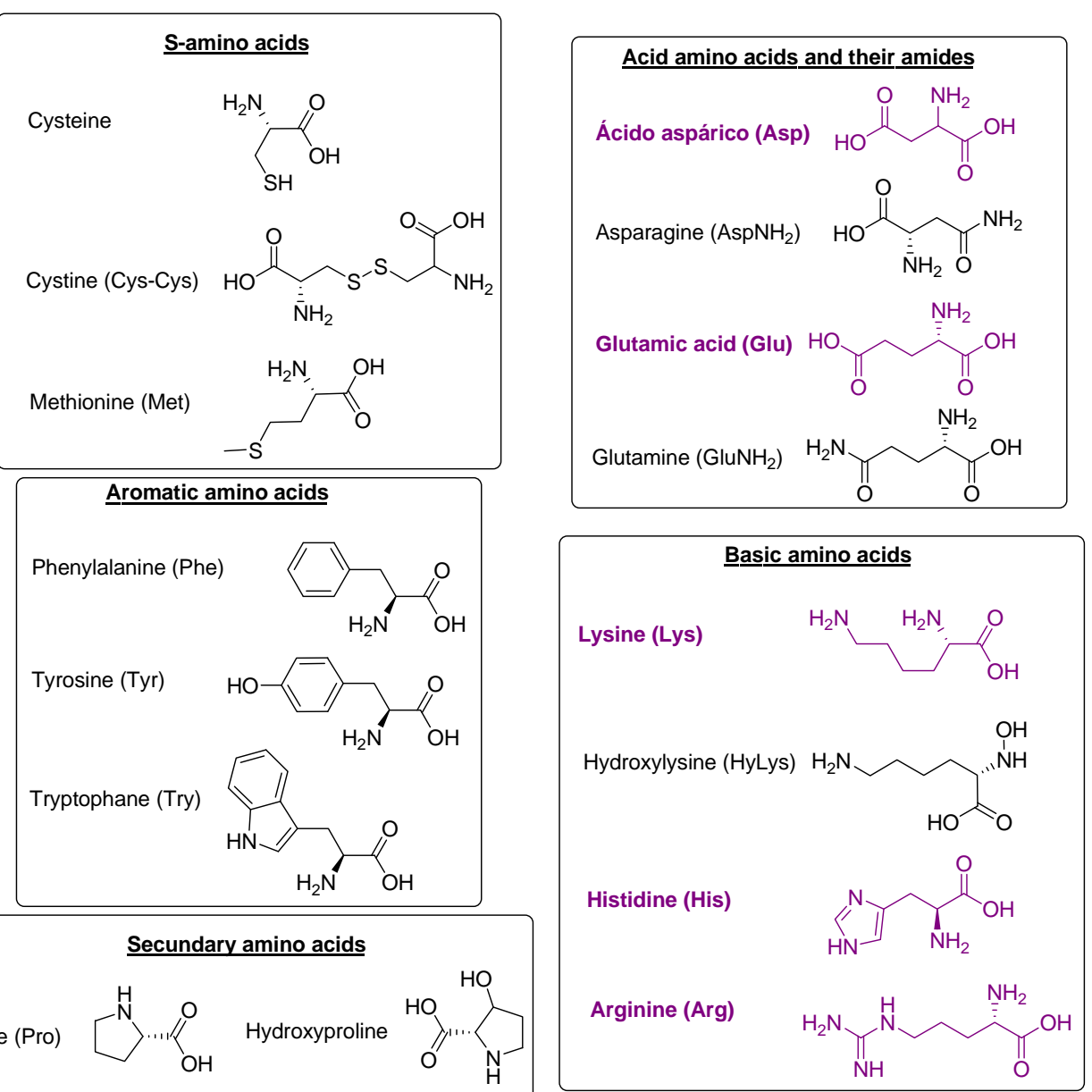


\section{Hydantoins}

Ferris et al. obtained hydantoin, 5,5-dimethyl-hydantoin and 5-carboxymethylidenehydantoin (Figure 2) using aqueous solutions of $\mathrm{NaCN}(1 \mathrm{M})$ at $\mathrm{pH} 9.2$ (adjusted with $\mathrm{HCl}$ ) with reaction times of several months at room temperature and after acid hydrolysis [81,85]. These compounds were identified using paper chromatography, thin layer chromatography and colorimetric methods.

These hydantoins are the cyclic products of the amino acids glycine, $\alpha$-aminoisobutyric acid and diaminosuccinic acid (Table 1). One possible pathway for the formation of these hydantoins during acid hydrolysis is the cyclisation of the respective carbamyl amino acids. The isolation of urea from the oligomerisation mixture and citrulline (Table 1) from the acid hydrolysate of the HCN oligomers is consistent with the presence of carbamyl groups in the HCN oligomers [80,86].

Figure 2. Hydantoins identified in the acid hydrolysate of the HCN oligomers.

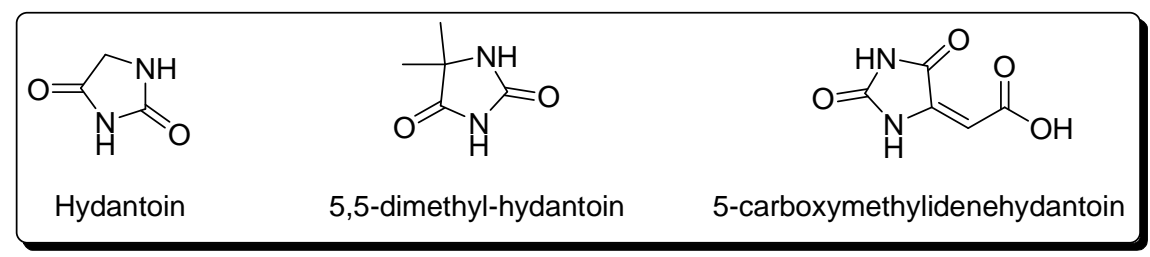

\section{Nucleic-Acid Bases}

It is generally accepted that one of the principal characteristic of life is the ability to transfer information from one generation to the next. All modern living organisms have a genetic code for storing and transmitting information based on a chemical system of nucleic acids: DNA and RNA. The nucleic acids contain the keys to construct the enzymes via the process of protein synthesis. Since Gilbert's hypothesis of an "RNA world" evolving at an early stage of life on primitive Earth, a variety of prebiotic syntheses have been proposed for the formation of the elementary building blocks of RNA and DNA: purine and pyrimine nucleic acid bases, ribose or 2'-deoxyribose, and phosphate [87,88]. Figure 3 shows the purines and pyrimidines present in the RNA and DNA (uracil in RNA and thymine in DNA). Today, it is well known that both RNA and DNA are able to carry out the catalytic activities required to start life on Earth $[89,90]$.

Figure 3. Nucleic-acid bases.
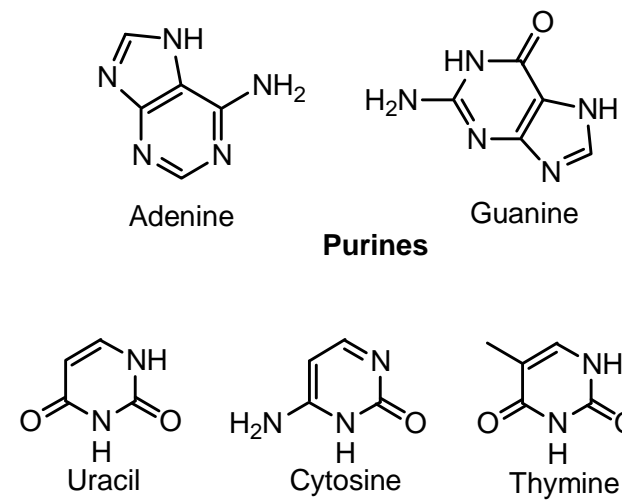

Pyrimidines

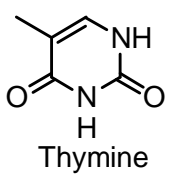




\subsection{Purines}

The first prebiotic synthesis of purines was carried out by Oró [6] using concentrated aqueous solutions of ammonium cyanide, which were heating at $70^{\circ} \mathrm{C}$ for several days. Adenine was identified in the raw reaction mixtures by the $\mathrm{R}_{\mathrm{f}}$ values (paper chromatography) and the UV spectra against authentic standards. Pullman showed that the purine base adenine occupies a unique position in the purine family: in comparison to the other purines, it has the greatest resonance energy per $\pi$-electron and thus is likely to have been incorporated preferentially into biomolecules [91].

Two years after the first detection of adenine in Oró's synthesis, Lowe et al. [77] also identified adenine and hypoxanthine in the total hydrolysed mixture from the heating of ammonium cyanide solution at $90{ }^{\circ} \mathrm{C}$ for $18 \mathrm{~h}$. During the next decade, Ferris and co-workers obtained adenine using diluted solution of $\mathrm{HCN}(0.1 \mathrm{M})$ adjusted to $\mathrm{pH} 9.2$ with $\mathrm{NH}_{4} \mathrm{OH}$ at room temperature for longer reaction times (4-12 months). Schwartz et al. explored in the 1980s the previous proposal made by Sanchez et al. [64,92]. Their results suggested that freezing dilute solutions of HCN in water could have provided conditions for the synthesis of bio-organic compounds in the primitive Earth. A freezing solution of $\mathrm{HCN}(0.01 \mathrm{M})$ was kept at $-2{ }^{\circ} \mathrm{C}$ for $98 \mathrm{~d}$ (initial $\mathrm{pH} 9.2$ adjusted with $\mathrm{NH}_{4} \mathrm{OH}$ ). The acid hydrolysate yielded $0.004 \%$ adenine [93]. The addition of glyconitrile (the product of the addition of $\mathrm{HCN}$ and formaldehyde) to the reaction mixture under the same reaction conditions increased the yield of adenine to $0.02 \%$. Indeed, Schwartz et al. demonstrated that formaldehyde, if introduced into solutions of $\mathrm{HCN}$, could accelerate the oligomerisation of $\mathrm{HCN}$ under certain conditions [94]. The same research group demonstrated that unhydrolysed oligomerisation mixtures prepared from $\mathrm{HCN}$ in the absence of formaldehyde contain adenine-8-carboxamide, a possible precursor of adenine [95]. However, 8-hydroxymethyladenine is mostly formed in oligomerisation solutions of HCN when formaldehyde is added, rather than adenine-8-carboxamide or adenine itself [96]. Also in the context of a primitive cold Earth or icy satellites, Miller and co-workers demonstrated the formation of several purines (xanthine, hypoxanthine, adenine, guanine and 2,6-diaminopurine) in oligomerisation processes of $\mathrm{HCN}$ at temperatures between -20 and $-78^{\circ} \mathrm{C}[66,67]$.

Adenine was also tentatively identified in radiolysis experiments with a pure aqueous solution of $\mathrm{HCN}$ (pH 6) [97].

All purines detected in $\mathrm{HCN}$ polymers are shown in Table 2, together with the cyanide polymerisation reaction conditions and the analytical tools used for their identification. Interestingly, the hydrolysis conditions significantly influence the yields for each purine found. In this context, Ferris et al. [82], Miyakawa et al. [66] and Borquez et al. [98] reported interesting works about the influence of the hydrolysis conditions on the yield of purines and other organics released from HCN polymers.

Both AMN and DAMN were independently detected and isolated by Matthews and Moser [25] and by Sánchez et al. [64]. 
Table 2. Purines identified in Hydrogen Cyanide (HCN) polymers. The reaction conditions are described together with the material analysed: soluble fraction (solution or soluble oligomers), insoluble fraction (precipitated dark solid) or a combination of both. $\mathrm{c}(\mathrm{M})=$ initial molar concentration of the reactant in aqueous solution; $\mathrm{HCN}_{(\mathrm{L})}=\mathrm{HCN}$ in liquid phase; $\mathrm{HCN}_{(\mathrm{G})}=\mathrm{HCN}$ in gas phase; $\mathrm{d}=$ days; $\mathrm{m}=\mathrm{moths} ; \mathrm{y}=\mathrm{years}$; (s) = saturated; Non hydrolysis = no additional hydrolysis (acid, basic or neutral) was made over the final product analysed; $\mathrm{Acid}=\mathrm{HCl}$ $6 \mathrm{~N} / 110{ }^{\circ} \mathrm{C} / 24 \mathrm{~h}$; Acid (2) $=\mathrm{HCl} 5 \mathrm{M} / 110{ }^{\circ} \mathrm{C} / 18 \mathrm{~h}$; Acid (3) $=98 \% \mathrm{HCOOH} / 170{ }^{\circ} \mathrm{C} / 2 \mathrm{~h}$; Neutral = phosphate $0.1 \mathrm{M}\left(\mathrm{pH}\right.$ 8)/140 ${ }^{\circ} \mathrm{C} / 3 \mathrm{days}$. Neutral $(2)=\mathrm{HCl}(\mathrm{pH} 8.5) / 110{ }^{\circ} \mathrm{C} / 24 \mathrm{~h}$; GC-MS = Gas Chromatography-Mass Spectrometry. PC = paper chromatography. TLC $=$ thin layer chromatography. $\mathrm{PE}=$ paper electrophoresis; HPLC-UV = High performance liquid chromatography-UV detector; D = detected but not quantified; $\mathrm{t}=$ tentatively identified.

\begin{tabular}{|c|c|c|c|c|c|c|c|}
\hline Compound & Starting material, c (M) & $\mathrm{T}\left({ }^{\circ} \mathrm{C}\right) / \mathrm{t} /$ Catalyser & Final product analysed & Hydrolysis & Yield (\%) & Method of identification & Reference \\
\hline \multirow{2}{*}{ Xhanthine } & \multirow{2}{*}{$\mathrm{HCN}, 0.1$} & \multirow{2}{*}{$-78 / 27 \mathrm{y} / \mathrm{NH}_{3}(\mathrm{pH} 9.2)$} & \multirow{2}{*}{ Solution + black solid } & Acid & 0.022 & \multirow{2}{*}{ HPLC-UV, GC-MS } & \multirow{2}{*}[66]{} \\
\hline & & & & Neutral & 0.022 & & \\
\hline \multirow[t]{4}{*}{ Hypoxanthine } & $\mathrm{HCN}, 1.5$ & $90 / 18 \mathrm{~h} / \mathrm{NH}_{3}$ & Solution + black solid & Acid & $\sim 1 \mu \mathrm{mol} / \mathrm{L}$ & PC, UV-spectrum, PE & [77] \\
\hline & $\mathrm{NaCN}, 1$ & $38 / 3-30 \mathrm{~d} / \mathrm{NH}_{4} \mathrm{Cl}$ & Black solid & Acid & $\mathrm{D}$ & GC-MS & {$[46]$} \\
\hline & & & Colution thlolk olid & Acid & 0.0041 & UPI $C$ UIV $C$ C MS & {$[66]$} \\
\hline & $\mathrm{HCN}, 0.1$ & $-78 / 27 \mathrm{y} / \mathrm{NH}_{3}(\mathrm{pH} 9.2)$ & Solution + black solid & Neutral & 0.0058 & HPLC-UV, GC-MS & [66] \\
\hline \multirow[t]{16}{*}{ Adenine } & $\mathrm{HCN},(\mathrm{s})$ & $90 / 24 \mathrm{~h} / \mathrm{NH}_{4} \mathrm{OH}(1.5 \mathrm{M})$ & Solution & Acid & $\mathrm{D}$ & PC, UV-spectrum & [6] \\
\hline & $\mathrm{HCN}, 1.5$ & $90 / 18 \mathrm{~h} / \mathrm{NH}_{3}$ & Solution + black solid & Acid & $\sim 1 \mu \mathrm{mol} / \mathrm{L}$ & PC, UV-spectrum, PE & [77] \\
\hline & HCN, 9.9 & $90 / 8 \mathrm{~d} / \mathrm{NH}_{4} \mathrm{OH}$ & Solution & Non hydrolysis & $60 \mathrm{mg} / \mathrm{L}$ & 2D-PC, UV-spectrum & [78] \\
\hline & $\mathrm{HCN}, 10$ & $80 / 24 \mathrm{~h} / \mathrm{NH}_{4} \mathrm{OH}$ & Solution & Acid & 0.027 & HPLC-UV & [99] \\
\hline & $\mathrm{HCN}, 1.5$ & $70 / 2 \mathrm{~d} / \mathrm{NH} 4 \mathrm{OH}(3 \mathrm{M})$ & Solution & Non hydrolysis & $\mathrm{D}$ & 2D-PC, UV-spectrum & [78] \\
\hline & & & & Non hydrolysis & $110 \mathrm{mg} / \mathrm{L}$ & & \\
\hline & $\mathrm{HCN}, 11.1$ & $70 / 5 \mathrm{~d} / \mathrm{NH}_{4} \mathrm{OH}(12.8 \mathrm{M})$ & Solution & Acid & $700 \mathrm{mg} / \mathrm{L}$ & 2D-PC, UV-spectrum & [78] \\
\hline & $\mathrm{NaCN}, 1$ & $38 / 3-30 \mathrm{~d} / \mathrm{NH}_{4} \mathrm{Cl}$ & Black solid & Acid & $\mathrm{D}$ & GC-MS & {$[46]$} \\
\hline & $\mathrm{HCN}, 14.6$ & r.t. $/ 26 \mathrm{~h} / \mathrm{NH}_{4} \mathrm{OH}(7 \mathrm{M})$ & Solution & Non hydrolysis & D & 2D-PC, UV-spectrum & [78] \\
\hline & $\mathrm{HCN}, 8.25$ & r.t. $/ 26 \mathrm{~h} / \mathrm{NH}_{4} \mathrm{OH}(13 \mathrm{M})$ & Solution & Non hydrolysis & $\mathrm{D}$ & 2D-PC, UV-spectrum & [78] \\
\hline & $\mathrm{HCN}, 0.1$ & r.t./1 w/ $/ \mathrm{NH}_{4} \mathrm{OH}(\mathrm{pH} 9.2)$ & Solution + black solid & Acid & 0.000013 & HPLC-UV, GC-MS & [66] \\
\hline & $\mathrm{HCN}, 0.1$ & r.t. $/ 4 \mathrm{w} / \mathrm{NH}_{4} \mathrm{OH}(\mathrm{pH} 9.2)$ & Solution + black solid & Acid & 0.00031 & HPLC-UV, GC-MS & [66] \\
\hline & $\mathrm{HCN}, 0.1$ & r.t./8 w/ $\mathrm{NH}_{4} \mathrm{OH}(\mathrm{pH} 9.2)$ & Solution + black solid & Acid & 0.00062 & HPLC-UV, GC-MS & [66] \\
\hline & $\mathrm{HCN}, 0.1$ & r.t. $/ 4-12 \mathrm{~m} / \mathrm{NH}_{4} \mathrm{OH}(\mathrm{pH} 9.2)$ & Soluble oligomers & Acid & $0.003-0.004$ & GC-MS & [82] \\
\hline & $\mathrm{NaCN}, 1$ & r.t./1 y/pH $9.2(\mathrm{HCl})$ & Soluble oligomers & Acid & $\mathrm{D}$ & TLC, UV-spectrum & [82] \\
\hline & $\mathrm{NaCN}, 2(+\mathrm{HCOH})$ & r.t./9 m/pH $9.2(\mathrm{HCl})$ & & $\begin{array}{c}\text { Non hydrolysis } \\
\text { Neutral (2) }\end{array}$ & $\begin{array}{c}3 \mu \mathrm{mol} / \mathrm{L} \\
0.06\end{array}$ & HPLC, UV spectrum, MS & [96] \\
\hline
\end{tabular}


Table 2. Cont

\begin{tabular}{|c|c|c|c|c|c|c|c|}
\hline Compound & Starting material, c (M) & $\mathrm{T}\left({ }^{\circ} \mathrm{C}\right) / \mathrm{t} /$ Catalyser & Final product analysed & Hydrolysis & Yield (\%) & Method of identification & Reference \\
\hline & $\mathrm{HCN}, 0.2$ & r.t. $-40^{\circ} \mathrm{C} / \gamma$-radiation $/ \mathrm{pH} 6$ & Solution & Acid (3) & $t$ & HPLC, GC-MS & {$[97]$} \\
\hline & HCN, 0.01 (+glyconitrile) & $-2 / 60 \mathrm{~d} / \mathrm{NH}_{4} \mathrm{OH}(\mathrm{pH} 9.2)$ & Solution & Acid (2) & 0.02 & HPLC-UV & [93] \\
\hline & $\mathrm{HCN}, 0.01$ & $-2 / 98 \mathrm{~d} / \mathrm{NH}_{4} \mathrm{OH}(\mathrm{pH} 9.2)$ & Solution & Acid (2) & 0.004 & HPLC-UV & [93] \\
\hline & $\mathrm{HCN}, 0.1$ & $-20 / 2 \mathrm{~m} / \mathrm{NH}_{3}(\mathrm{pH} 9.2)$ & Solution & Acid & 0.005 & HPLC-UV, ESI-MS & {$[67]$} \\
\hline & $\mathrm{HCN}, 0.001$ & $-20 / 3 \mathrm{~m} / \mathrm{NH}_{4} \mathrm{OH}(\mathrm{pH} 9.2)$ & Solution + black solid & Acid & 0.0042 & HPLC-UV, GC-MS & {$[66]$} \\
\hline & $\mathrm{HCN}, 0.01$ & $-20 / 3 \mathrm{~m} / \mathrm{NH}_{4} \mathrm{OH}(\mathrm{pH} 9.2)$ & Solution + black solid & Acid & 0.01 & HPLC-UV, GC-MS & {$[66]$} \\
\hline & $\mathrm{HCN}$ & $-20 / 3 \mathrm{~m} / \mathrm{NH}_{4} \mathrm{OH}(\mathrm{pH} 9.2)$ & Solution + black solid & Acid & 0.0094 & HPLC-UV, GC-MS & {$[66]$} \\
\hline & $\mathrm{HCN}, 0.1$ & $-20 / 25 \mathrm{y} / \mathrm{NH}_{3}(\mathrm{pH} 9.2)$ & Solution & Acid & 0.035 & HPLC-UV, ESI-MS & {$[67]$} \\
\hline & $\mathrm{NaCN}, 0.1$ & $-30 / 2 \mathrm{~m} / \mathrm{NH}_{4} \mathrm{Cl}$ & Solution & Acid & 0.0004 & HPLC-UV & [99] \\
\hline & $\mathrm{HCN}, 0.1$ & $-78 / 25 \mathrm{y} / \mathrm{NH}_{3}(\mathrm{pH} 9.2)$ & Solution & Acid & 0.04 & HPLC-UV, ESI-MS & {$[67]$} \\
\hline & & & & Acid & 0.029 & & \\
\hline & $\mathrm{HCN}, 0.1$ & $-78 / 27 \mathrm{y} / \mathrm{NH}_{3}(\mathrm{pH} 9.2)$ & Solution + black solid & Neutral & 0.012 & HPLC-UV, GC-MS & {$[66]$} \\
\hline & & & & Non hydrolysis & 0.00016 & & \\
\hline \multirow[t]{7}{*}{ Guanine } & $\mathrm{HCN}, 10$ & $80 / 24 \mathrm{~h} / \mathrm{NH}_{4} \mathrm{OH}$ & Solution & Acid & 0.0007 & HPLC-UV & [99] \\
\hline & $\mathrm{NaCN}, 1$ & $38 / 3-30 \mathrm{~d} / \mathrm{NH}_{4} \mathrm{Cl}$ & Black solid & Acid & $\mathrm{D}$ & GC-MS & {$[46]$} \\
\hline & $\mathrm{HCN}, 0.1$ & $-20 / 25 \mathrm{y} / \mathrm{NH}_{3}(\mathrm{pH} 9.2)$ & Solution & Acid & 0.0004 & HPLC-UV, ESI-MS & {$[67]$} \\
\hline & $\mathrm{NaCN}, 0.1$ & $-30 / 2 \mathrm{~m} / \mathrm{NH}_{4} \mathrm{Cl}$ & Solution & Acid & 0.000014 & HPLC-UV & [99] \\
\hline & & & & Acid & 0.0067 & & \\
\hline & $\mathrm{HCN}, 0.1$ & $-78 / 27 \mathrm{y} / \mathrm{NH} 3(\mathrm{pH} 9.2)$ & Solution + black solid & Neutral & 0.0033 & HPLC-UV, GC-MS & {$[66]$} \\
\hline & & & & Non hydrolysis & 0.00011 & & \\
\hline 2,6-diaminopurine & $\mathrm{HCN}, 0.1$ & $-78 / 27$ y/NH3 (pH 9.2) & Solution + black solid & Neutral & 0.0091 & HPLC-UV, GC-MS & {$[66]$} \\
\hline 8-hydroxymethyladenine & $\mathrm{NaCN}, 2(+\mathrm{HCOH})$ & r.t. $/ 9 \mathrm{~m} / \mathrm{pH} 9.2(\mathrm{HCl})$ & & $\begin{array}{c}\text { Non hydrolysis } \\
\text { Neutral (2) }\end{array}$ & $\begin{array}{c}47 \mu \mathrm{mol} / \mathrm{L} \\
0.06 \\
\end{array}$ & HPLC, UV spectrum, MS & [96] \\
\hline
\end{tabular}


Oró proposed that the formation of adenine proceeded by the reaction of the HCN trimer (aminomalonitrile, AMN) with formamidine [78]. However, this mechanism was discarded because the hydrolysis of formamidine is very rapid and because the relative rate of reaction of cyanide with AMN is much greater than the rate of the reaction of formamide with AMN [64]. These results suggested that the conditions required for the formation of AMN will result in its rapid conversion to the tetramer (diaminomalonitrile, DAMN) by reaction with cyanide. Thus, DAMN or compounds derived from it must be precursors of adenine. This mechanistic proposal by Ferris and Orgel is shown in Figure 4a: AMN reacts to formamide to produce 4[5]-aminoimidazole-5[4]-carbonitrile (AICN), a compound that readily yields a variety of purines under plausible prebiotic conditions [100,101] This synthesis is not possible, as it is indicated above, in dilute solutions because of the competing hydrolysis of formamidine to formamide. On the other hand, independently of the formamidine concentration, Ferris and Orgel demonstrated the photochemical rearrangement of DAMN into AICN [100]. AICN and a product of its hydrolysis, 4[5]-aminoimidazole-5[4]-carboxamide (AICA), reacts with $\mathrm{HCN}$, cyanate $\left(\mathrm{NCO}^{\top}\right)$, formamidine, or cyanogen $(\mathrm{CN})_{2}$ to give adenine, hypoxanthine, diaminopurine, xanthine, isoguanine and guanine, respectively. Both imidazoles, AICA and AICN have been identified in HCN polymers [78,82].

Schwartz and Voet proposed a pathway from HCN to adenine that does not implicate AICN or AICA as an intermediate (Figure 4b). This mechanism is based on the isolation of adenine-8-carboxamide, which readily hydrolyses to adenine [95]. In this approach, the main precursor is also DAMN, a compound that is stable and present in appreciable concentration in an oligomerising solution of HCN [58]. The 2-substituted imidazole derivatives 2-cyano-AICA, 4[5]-aminoimidazole-2,5[4]-dicarboxamide (AIDCA) and 4[5]- $\mathrm{N}$-(aminomethylidene)-aminoimidazole-2,5[4]-dicarboxamide (AMAIDCA) were isolated from $\mathrm{HCN}$ polymers using a solution of $\mathrm{HCN}\left(1 \mathrm{M}, \mathrm{pH} 9.2\right.$ adjusted with $\mathrm{NH}_{4} \mathrm{OH}$ ) stored at room temperature for eight months. The 2-substituted imidazoles were identified before hydrolysis using spectroscopic techniques and mass spectrometry [95].

Figure 4. Proposed mechanisms for the formation of purines from HCN. (a) Ferris and Orgel; (b) Voet and Schwartz. AICA, 4[5]-aminoimidazole-5[4]-carboxamide; AICN, 4[5]-aminoimidazole-5[4]-carbonitrile; AIDCA, 4[5]-aminoimidazole-2,5[4]-dicarboxamide; AMAIDCA, 4[5]- $\mathrm{N}$-(aminomethylidene)-aminoimidazole-2,5[4]-dicarboxamide; Adenine-8-ca, adenine-8-carboxamide. The purines and their precursors identified in the HCN polymers are marked in purple.

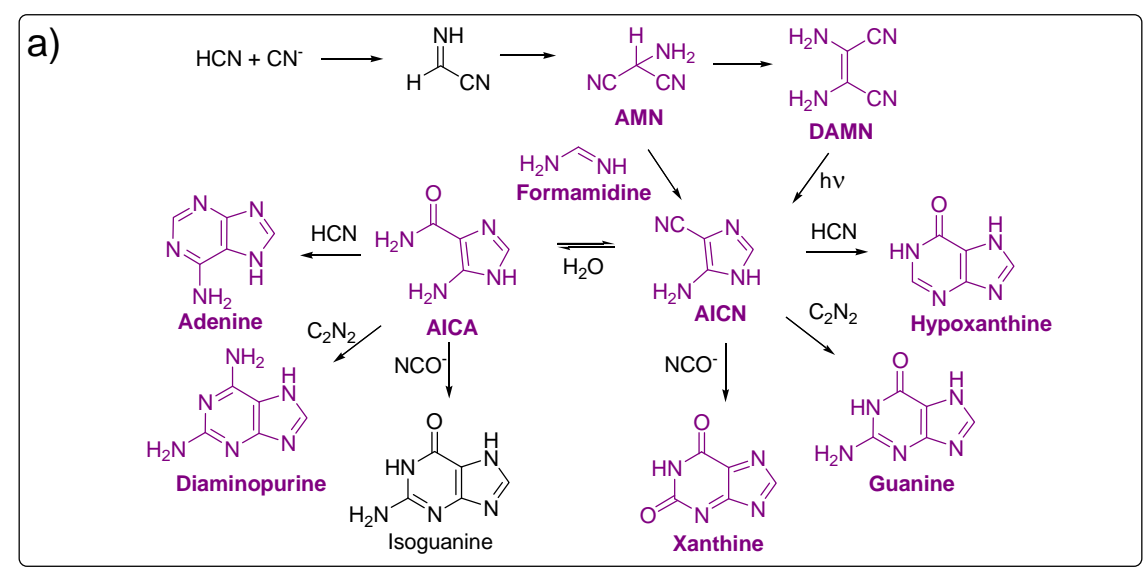


Figure 4. Cont.

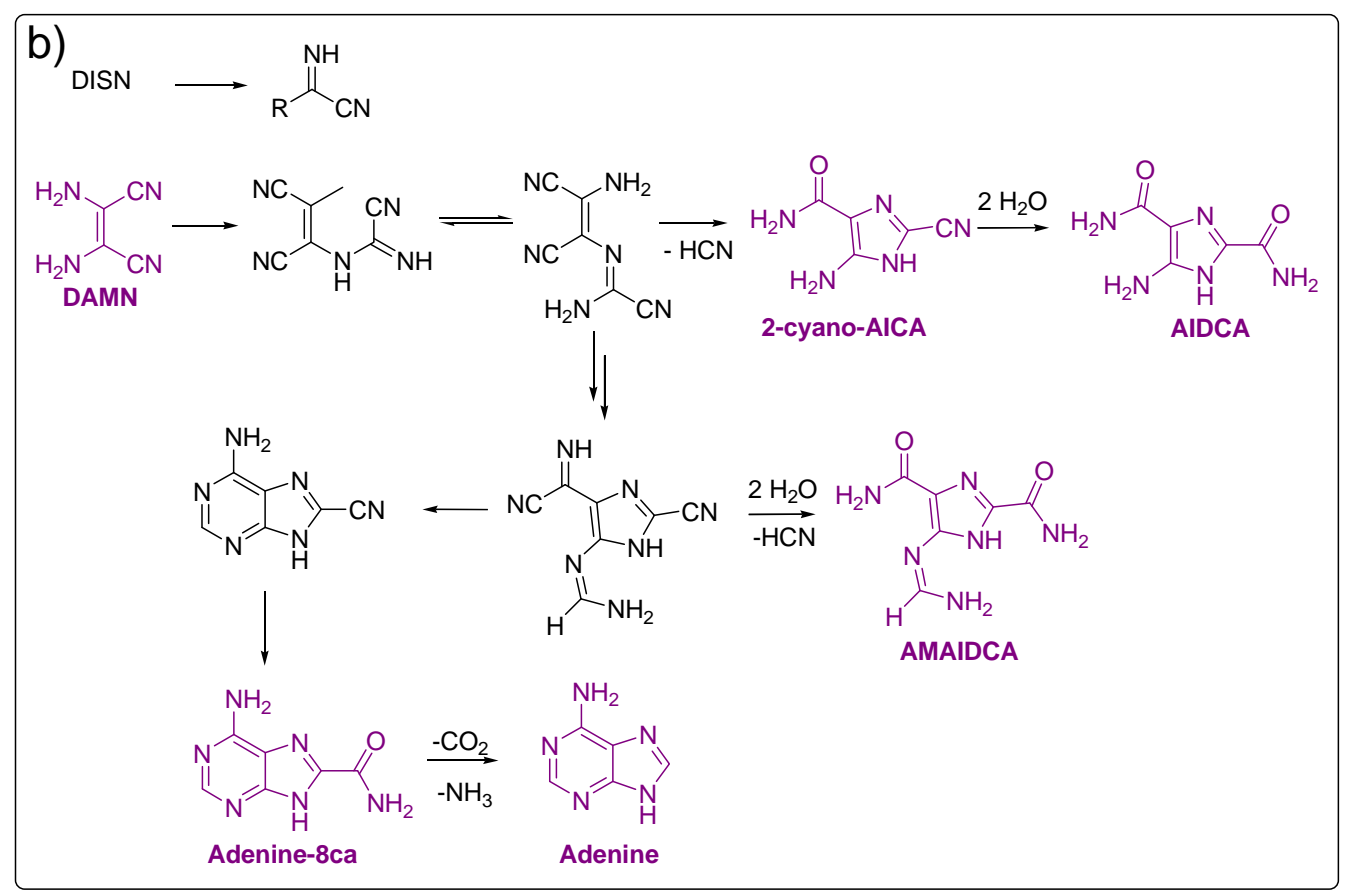

\subsection{Pyrimidines}

In the late 1970s, Ferris and co-worker identified pyrimidines for the first time (4,5-dihydroxypyrimidine, 5-hydroxyuracil and orotic acid) in the acid hydrolysates of HCN oligomers synthesised at room temperature [82,102]. Under similar polymerisation conditions for $\mathrm{HCN}$, Voet and Schwartz identified uracil using a laborious purification work-up and hydrolysing HCN oligomers [103]. Under the simulation conditions of a cold Earth, Miyakawa et al. achieved the greatest diversity in pyrimidines (4,5-dihydroxypyridine, uracil, 5-hydroxyuracil, 5-aminouracil, orotic acid and 5-aminoorotic acid) [66]. Tentatively, Negrón-Mendoza et al. detected uracil, cytosine and thymine in experiments of radiolysis of $\mathrm{HCN}$ aqueous solutions [97]. In Table 3, all pyrimidines found in $\mathrm{HCN}$ polymers are summarised. As in the case of purines, the yield in pyrimidines released from HCN polymers depends strongly on the hydrolysis conditions assayed (see Table 3). Additionally, it is interesting to note that the development of analytical chromatography techniques has allowed the identification of a greater diversity of pyrimidines, as can be readily seen in the more recent works [46,66].

Ferris et al. proposed the following mechanism (Figure 5) to explain the formation of the pyrimidines identified by them, starting from the stable tetramer of HCN, DAMN [102]. 
Table 3. Pyrimides identified in HCN polymers. The reaction conditions are described together with the material analysed: soluble fraction (solution or soluble oligomers), insoluble fraction (precipitated dark solid) or a combination of both. $\mathrm{c}(\mathrm{M})=$ initial molar concentration of the reactant in aqueous solution; $\mathrm{d}=$ days; $\mathrm{m}=$ moths; $\mathrm{y}=$ years; Acid $=\mathrm{HCl} 6 \mathrm{~N} / 110^{\circ} \mathrm{C} / 24 \mathrm{~h}$; Non hydrolysis = no additional hydrolysis was made over the final product analysed; Acid (2) $=\mathrm{HCl} 5 \mathrm{M} / 110{ }^{\circ} \mathrm{C} / 18 \mathrm{~h}$; Acid $(3)=98 \% \mathrm{HCOOH} / 170{ }^{\circ} \mathrm{C} / 2 \mathrm{~h}$; Neutral = phosphate $0.01 \mathrm{M}(\mathrm{pH} 8) / 140{ }^{\circ} \mathrm{C} / 3$ days; Neutral (2) $=\mathrm{NaOH}(\mathrm{pH} 5) / 110{ }^{\circ} \mathrm{C} / 24$ h; GC-MS = Gas Chromatography-Mass Spectrometry; TLC = thin layer chromatography; HPLC-UV = High performance liquid chromatography-ultraviolet detector; $\mathrm{D}=$ detected but not quantified; $\mathrm{t}=$ entatively identified.

\begin{tabular}{|c|c|c|c|c|c|c|c|}
\hline Compound & Starting material, c(M) & $\mathrm{T}\left({ }^{\circ} \mathrm{C}\right) / \mathrm{t} /$ Catalyst & Final product analysed & Hydrolysis & Yield (\%) & Method of identification & Reference \\
\hline 4,5-Dihydroxypyrimidine & $\mathrm{HCN}, 0.1$ & r.t./4-12 m/ $/ \mathrm{NH}_{4} \mathrm{OH}(\mathrm{pH} 9.2)$ & Soluble oligomers & Acid & 0.62 & TLC, UV spectrum & {$[102]$} \\
\hline \multirow{8}{*}{ Uracil } & $\mathrm{HCN}, 0.1$ & r.t. $/ 4-12 \mathrm{~m} / \mathrm{NH}_{4} \mathrm{OH}(\mathrm{pH} 9.2$ ) & Soluble oligomers & Acid & $0.7-0.9$ & GC-MS & {$[82]$} \\
\hline & $\mathrm{NaCN}, 1$ & r.t./1 y/pH $9.2(\mathrm{HCl})$ & Soluble oligomers & Acid & $\mathrm{D}$ & TLC, UV spectrum & [82] \\
\hline & $\mathrm{HCN}, 0.1$ & $-78 / 27 \mathrm{y} / \mathrm{NH}_{3}(\mathrm{pH} 9.2)$ & Solution + black solid & Acid & 0.65 & HPLC-UV, GC-MS & [66] \\
\hline & $\mathrm{NaCN}, 1$ & $38 / 3-30 \mathrm{~d} / \mathrm{NH}_{4} \mathrm{Cl}$ & Black solid & Acid & $\mathrm{D}$ & GC-MS & [46] \\
\hline & $\mathrm{HCN}, 1$ & r.t. $/ 6$ m/NH $\mathrm{NH}_{4} \mathrm{OH}(\mathrm{pH} 9.2)$ & Solution & Acid (2) & 0.001 & HPLC-UV & [103] \\
\hline & $\mathrm{HCN}, 0.1$ & r.t./6 m/NH $\mathrm{NH}_{4} \mathrm{OH}(\mathrm{pH} 9.2)$ & Solution & Acid (2) & 0.005 & HPLC-UV & [103] \\
\hline & $\mathrm{NaCN}, 1$ & r.t./6 m/pH $9.2(\mathrm{HCl})$ & Solution & Acid (2) & 0.001 & HPLC-UV & [103] \\
\hline & $\mathrm{HCN}, 0.1$ & $-78 / 27 \mathrm{y} / \mathrm{NH}_{3}(\mathrm{pH} 9.2)$ & Solution + black solid & $\begin{array}{c}\text { Acid } \\
\text { Neutral }\end{array}$ & $\begin{array}{c}0.00026 \\
0.0017\end{array}$ & HPLC-UV, GC-MS & [66] \\
\hline \multirow{5}{*}{ 5-Hydroxyuracil } & $\mathrm{HCN}, 0.2$ & r.t. $-40^{\circ} \mathrm{C} / \gamma-\operatorname{rad}(60 \mathrm{Co}) / \mathrm{pH} 6$ & Solution & Acid (3) & $\mathrm{t}$ & HPLC, GC-MS & [97] \\
\hline & $\mathrm{NaCN}, 1$ & $38 / 3-30 \mathrm{~d} / \mathrm{NH}_{4} \mathrm{Cl}$ & Black solid & Acid & $\mathrm{D}$ & GC-MS & {$[46]$} \\
\hline & $\mathrm{HCN}, 0.1$ & r.t. $/ 4-12 \mathrm{~m} / \mathrm{NH}_{4} \mathrm{OH}(\mathrm{pH} 9.2)$ & Soluble oligomers & Acid & $0.002-0.004$ & TLC, UV spectrum & [102] \\
\hline & $\mathrm{HCN}, 0.1$ & r.t. $/ 4-12 \mathrm{~m} / \mathrm{NH}_{4} \mathrm{OH}(\mathrm{pH} 9.2)$ & Soluble oligomers & Acid & 0.003 & GC-MS & {$[82]$} \\
\hline & $\mathrm{HCN}, 0.1$ & $-78 / 27 \mathrm{y} / \mathrm{NH}_{3}(\mathrm{pH} 9.2)$ & Solution + black solid & Acid & 0.0015 & HPLC-UV, GC-MS & [66] \\
\hline Cytosine & $\mathrm{HCN}, 0.2$ & r.t. $-40{ }^{\circ} \mathrm{C} / \gamma-\operatorname{rad}\left({ }^{60} \mathrm{Co}\right) / \mathrm{pH} 6$ & Solution & Acid (3) & $\mathrm{t}$ & HPLC, GC-MS & [97] \\
\hline \multirow[t]{2}{*}{ 5-Aminouracil } & $\mathrm{NaCN}, 1$ & $38 / 3-30 \mathrm{~d} / \mathrm{NH}_{4} \mathrm{Cl}$ & Black solid & Acid & $\mathrm{D}$ & GC-MS & [46] \\
\hline & $\mathrm{HCN}, 0.1$ & $-78 / 27 \mathrm{y} / \mathrm{NH}_{3}(\mathrm{pH} 9.2)$ & Solution + black solid & $\begin{array}{c}\text { Acid } \\
\text { Neutral }\end{array}$ & $\begin{array}{l}0.0058 \\
0.0038\end{array}$ & HPLC-UV, GC-MS & [66] \\
\hline \multirow[t]{4}{*}{ Orotic acid } & $\mathrm{NaCN}, 1$ & $38 / 3-30 \mathrm{~d} / \mathrm{NH}_{4} \mathrm{Cl}$ & Black solid & Acid & $\mathrm{D}$ & GC-MS & {$[46]$} \\
\hline & $\mathrm{HCN}, 0.1$ & r.t./4-12 m/NH $/ \mathrm{NH}_{4} \mathrm{OH}(\mathrm{pH} 9.2)$ & Soluble oligomers & Neutral (2) & 0.009 & TLC, UV spectrum & [102] \\
\hline & $\mathrm{HCN}, 0.1$ & r.t./4-12 m/ $\mathrm{NH}_{4} \mathrm{OH}(\mathrm{pH} 9.2)$ & Soluble oligomers & Acid & 0.009 & GC-MS & {$[82]$} \\
\hline & $\mathrm{HCN}, 0.1$ & $-78 / 27$ y/NH $\mathrm{NH}_{3}(\mathrm{pH} 9.2)$ & Solution + black solid & $\begin{array}{c}\text { Acid } \\
\text { Neutral }\end{array}$ & $\begin{array}{c}0.0025 \\
0.1\end{array}$ & HPLC-UV, GC-MS & [66] \\
\hline \multirow[t]{2}{*}{ 5-Aminoorotic acid } & $\mathrm{HCN}, 0.1$ & $-78 / 27 \mathrm{y} / \mathrm{NH}_{3}(\mathrm{pH} 9.2)$ & Solution + black solid & Neutral & 0.019 & HPLC-UV, GC-MS & [66] \\
\hline & $\mathrm{HCN}, 0.1$ & $-78 / 27 \mathrm{y} / \mathrm{NH}_{3}(\mathrm{pH} 9.2)$ & Solution + black solid & Non hydrolysis & 0.00028 & HPLC-UV, GC-MS & [66] \\
\hline Thymine & $\mathrm{HCN}, 0.2$ & r.t. $-40{ }^{\circ} \mathrm{C} / \gamma-\operatorname{rad}\left({ }^{60} \mathrm{Co}\right) / \mathrm{pH} 6$ & Solution & Acid (3) & $\mathrm{t}$ & HPLC, GC-MS & [97] \\
\hline 1,2,5,6-Tetrahydropyrimidine & $\mathrm{NaCN}, 1$ & $38 / 3-30 \mathrm{~d} / \mathrm{NH}_{4} \mathrm{Cl}$ & Black solid & Acid & $\mathrm{D}$ & GC-MS & [46] \\
\hline
\end{tabular}


Figure 5. Proposal mechanisms for the formation of pyrimidines from $\mathrm{HCN}$ by Ferris et al. [102]. The compounds identified in the HCN polymers are marked in purple.

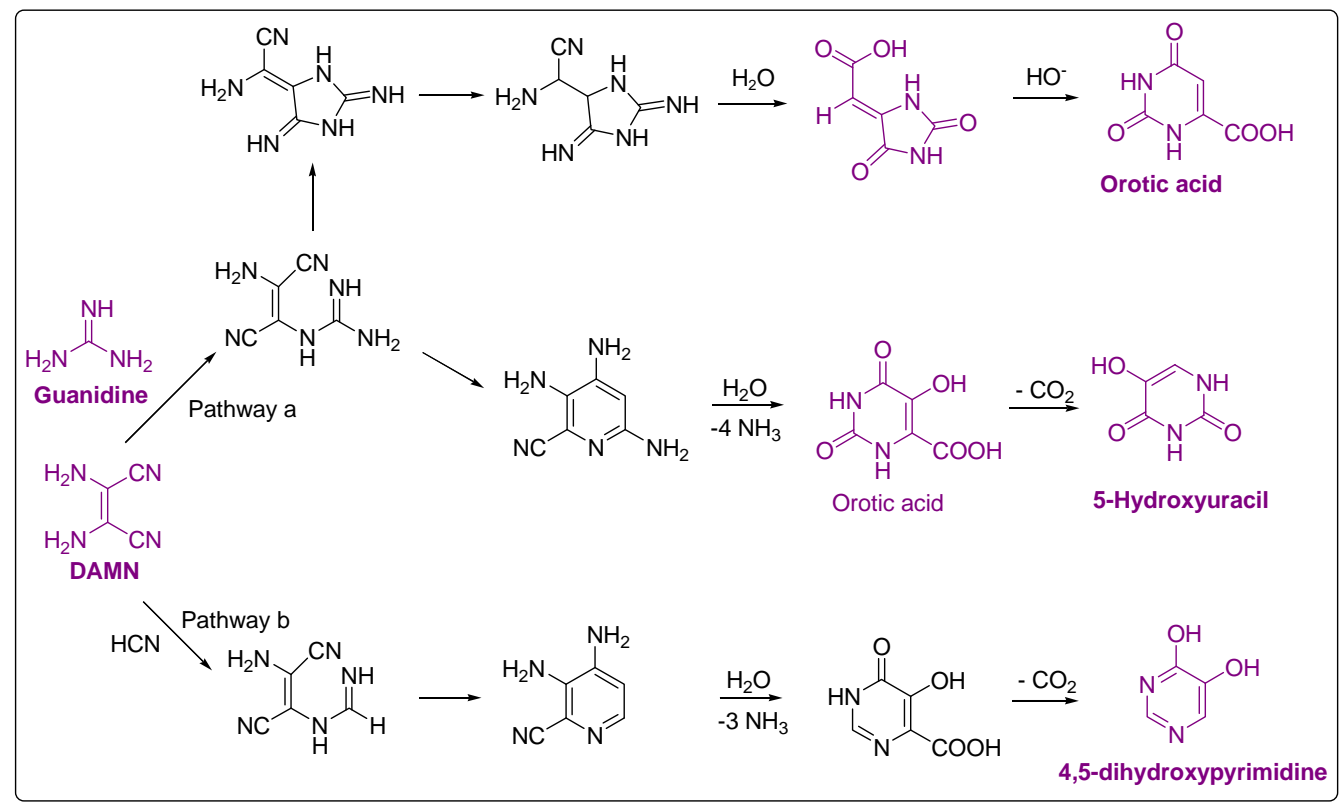

\section{Carboxylic Acids}

As we can see, the above HCN polymers contain the chemical information for the synthesis of nucleic-acid bases and have been used as macromolecular precursors for both purine and pyrimidine derivatives upon acidic or basic hydrolysis and as amino acids. Moreover, the HCN polymers also present the ability to release carboxylic acids after acid hydrolysis.

Several monocarboxylic acids have been detected in HCN polymers. Lowe et al. 1963 [77] identified formic acid in an ether extract of a dried portion of the total reaction mixture in an experiment at a high polymerisation temperature. In an experiment with moderate conditions, glycolic acid was identified using GC-MS in the black insoluble polymer, after acid hydrolysis [46]. The radiolysis of HCN using a 60Co source led to the production of butyric acid [104].

In this same radiolysis experiment, a large set of dicarboxylic and tricarboxylic acids were identified as their methyl-ester derivatives extracted with water-benzene using GC-MS [104] (Figure 6). This experiment was also reproduced in the presence of clays, and again a set of carboxylic acids was obtained [105]. The production of carboxylic acids is a function of the radiation dose, and the presence of clays led to lower production. The proposed mechanism for the formation of carboxylic acids in radiolysis experiments of $\mathrm{HCN}$ is the following:

\begin{tabular}{|c|c|c|}
\hline $\mathrm{RCN}+\cdot \mathrm{OH}$ & $\longrightarrow$ & $\mathrm{RC}(\mathrm{OH})=\mathrm{N}$ or $\mathrm{RC}=\mathrm{NOH}$ \\
\hline $\mathrm{RCN}+\cdot \mathrm{H}$ & $\longrightarrow$ & $\mathrm{RC}(\mathrm{H})=\mathrm{N}$ or $\mathrm{RC}=\mathrm{NH}$ \\
\hline $\mathrm{RCN}+\mathrm{e}_{\mathrm{aq}}$ & $\longrightarrow$ & $(\mathrm{RCN})^{-}$ \\
\hline$(\mathrm{RCN})^{-}+\mathrm{H}_{2} \mathrm{O}$ & $\longrightarrow$ & $\mathrm{RC}(\mathrm{H})=\mathrm{N}$ or $\mathrm{RC}=\mathrm{NH}$ \\
\hline $\mathrm{RCH}_{2} \mathrm{CN}+\cdot \mathrm{H} / \cdot \mathrm{OH}$ & $\longrightarrow$ & $\mathrm{RCHCN}+\mathrm{H}_{2} / \mathrm{H}_{2} \mathrm{O}$ \\
\hline $\mathrm{RCHCN}+\mathrm{H}_{2} \mathrm{O}$ & & $\mathrm{RCHCOOH}$ \\
\hline
\end{tabular}


Oxalic acid is largely known as a polymerisation product of $\mathrm{HCN}$ in aqueous solutions [81]. However, it has recently been demonstrated that the formation of other dicarboxylic acids is possible in NH4CN polymerisation processes. The acid hydrolysates of black HCN polymers yielded oxalic acid, malonic acid, 2-hydroxymalonic acid, succinic acid and maleic acid [46].

Figure 6. Di- and tricarboxylic acids produced by radiolysis of HCN. In bold are marked the acids also obtained from polymerisation of $\mathrm{NH}_{4} \mathrm{CN}$.

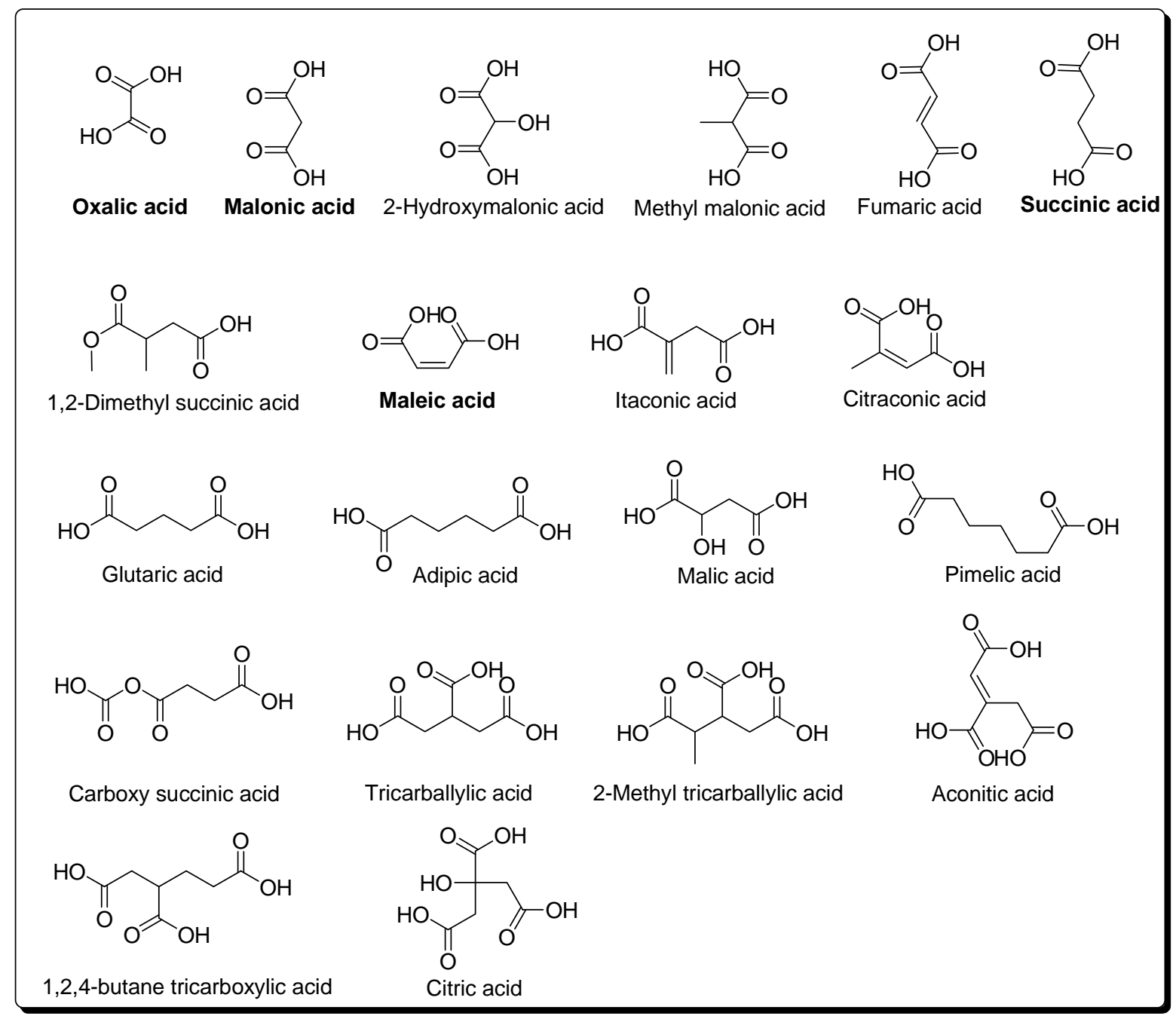

The identification of di- and tricarboxylic acids from HCN polymerisation is very interesting from the perspective of the emergence of a primordial metabolic cycle. Eschenmoser suggested that a relationship exists between $\mathrm{HCN}$ and the constituents of the reductive citric acid cycle [106]. The acids implicated in a plausible inverse Kreb's cycle and the di- and tricarboxylic acids identified in HCN polymers are shown in Figure 7.

\section{Carbonyl Compounds}

In the radiolysis experiments of aqueous solutions of $\mathrm{HCN}$ in the absence or presence of $\mathrm{NH} 3(\mathrm{pH} 6$ and 9, respectively), several aldehydes and ketones were identifiable in the crude reaction mixtures (Figure 8) using GC-FID [104]. 
Figure 7. The carboxylic acids detected in the HCN polymers that are related to a plausible reductive Krebs cycle are marked in purple. The scheme is adapted from Smith and Morowitz [107].

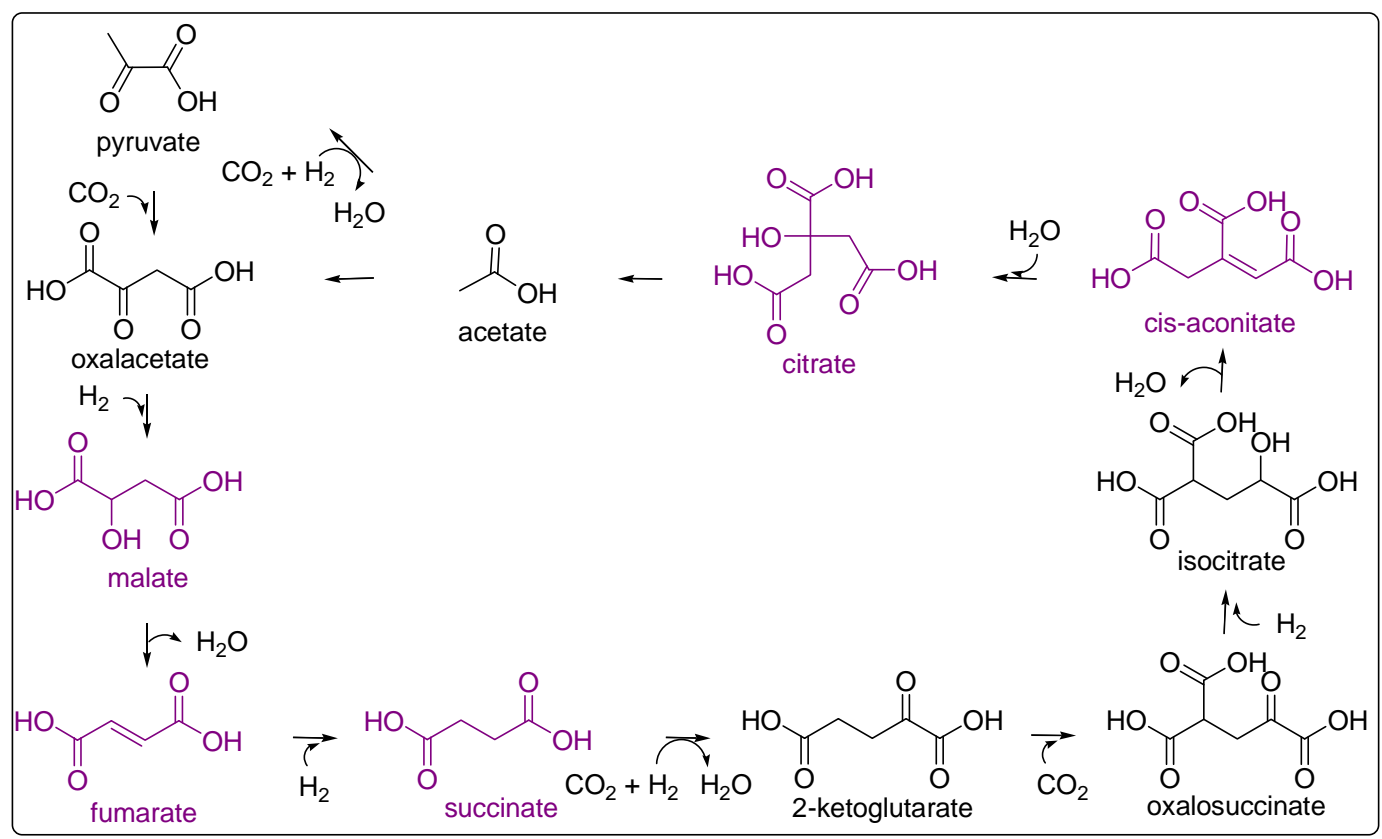

Figure 8. Aldehydes and ketones obtained in radiolysis experiments of aqueous HCN solutions.

\begin{tabular}{|cccc|}
\begin{tabular}{|llll}
0 \\
Formaldehyde
\end{tabular} & Acetaldehyde & Methylglioxal & Acetone \\
\hline
\end{tabular}

\section{Pteridines}

Recently, Ruiz-Bermejo et al. [21] identified a pteridine, 2,4,7-trihydroxy-pteridine, during preliminary analysis of the acid hydrolysate of black HCN polymers using GC-MS. These results are interesting because this compound can be related to several cofactors, such as riboflavins and pterins. Moreover, the cofactors or coenzyme structures are considered "molecular fossils" of an early phase of life, and a hydrocyanic origin of cofactor building blocks has been suggested [1,108-110].

\section{Others}

Urea is a principal product of the polymerisation reactions of $\mathrm{HCN}$ in aqueous solutions. It can be identified before hydrolysis of the crude reaction mixtures [72,77,81,82]. Lowe et al. [77] estimated a yield of $0.16 \mathrm{~mol} / \mathrm{L}$ in heating experiments for the polymerisation of $\mathrm{HCN}$. The hydrolysis product of urea, formamide, and formamidine can also be identified [78] together with other urea related compounds, such as guanidine [72,82], guanidinoacetic acid [72,82] and cyanamide [111] in HCN polymers. These compounds were identified by their $\mathrm{R}_{\mathrm{f}}$ values against authentic standards and by colorimetric methods (Figure 9). 
Figure 9. Urea and related compounds identified in the reaction mixtures of the HCN polymers.

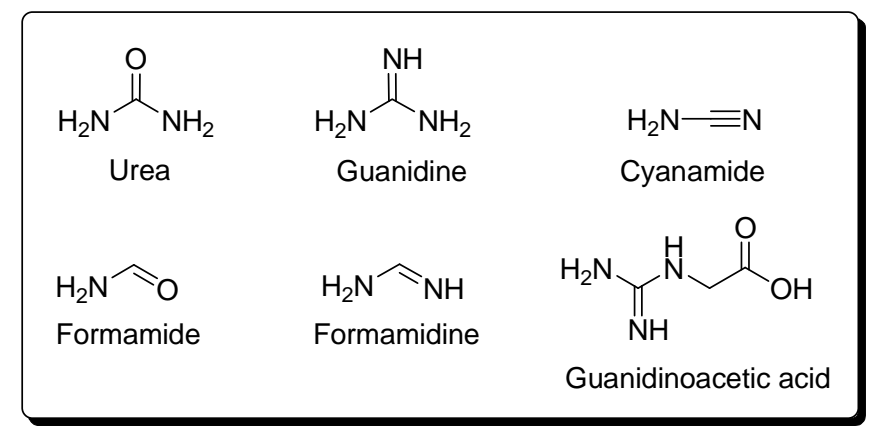

\section{Summary}

HCN polymers can be obtained under a wide variety of conditions. Depending on the polymerisation conditions, the treatment of the raw reactions and/or the analytical tools, a great diversity of simple organics and biomonomers can be detected. In HCN polymers, amino acids, purines, pyrimidines, carboxylic acids, aldehydes and ketones, pteridines, urea and urea-related compounds have been identified (Figure 10). Many of these organics compounds are building blocks of current proteins and nucleic acids or are active participants in metabolism. Therefore, the HCN polymers play a central core in a plausible protobiological system.

Figure 10. Summary of the simple organics and biomonomers identified in HCN polymers.

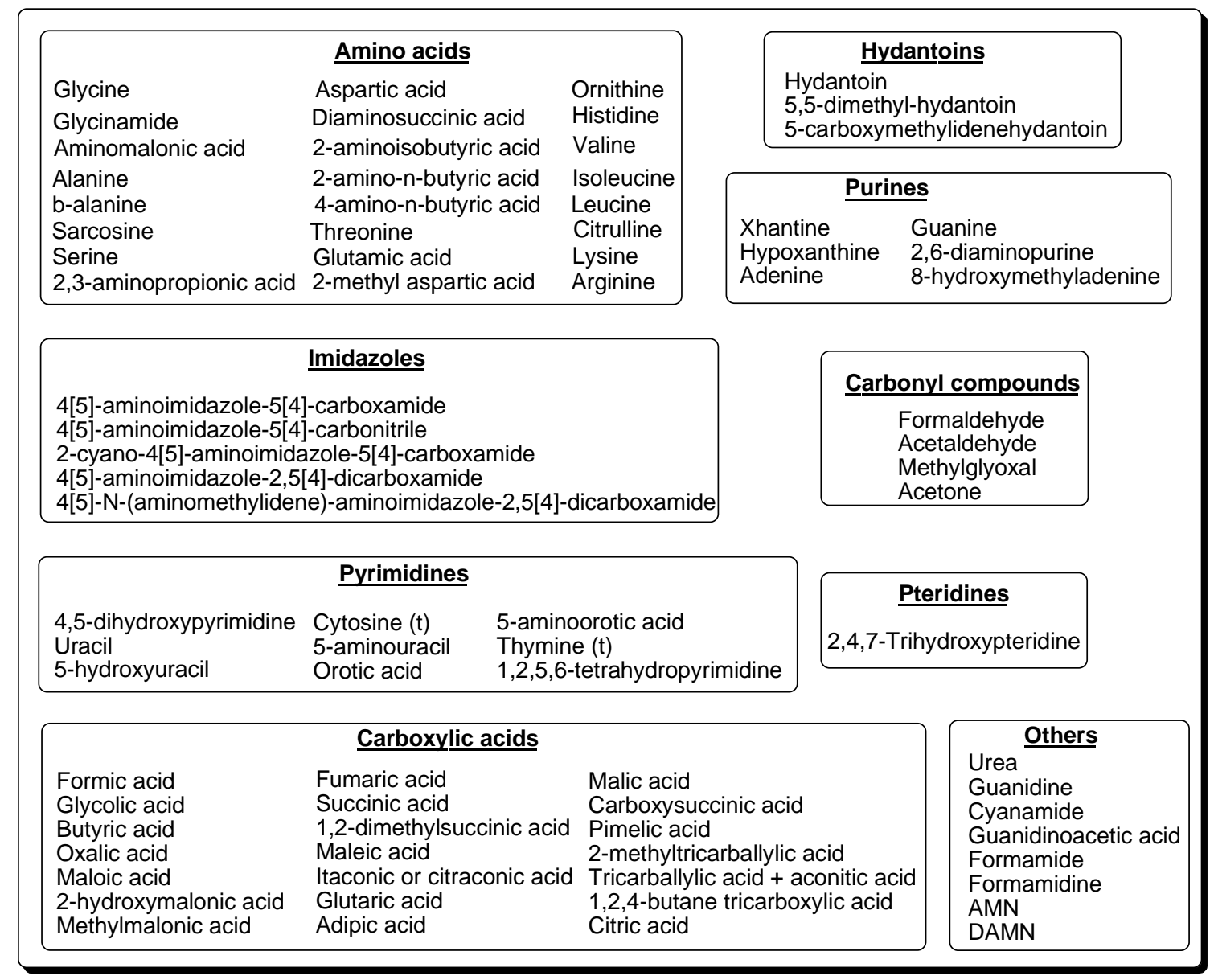




\section{Acknowledgments}

The authors used the research facilities of Centro de Astrobiología (CAB) and were supported by the Instituto Nacional de Técnica Aeroespacial "Esteban Terradas" (INTA) and the projects AYA2009-13920-C02-01 of the Ministerio de Ciencia e Innovación (Spain). Also we thank the support of the Economy and Competitivity Ministry (Project AYA2011-25720).

\section{References}

1. Miller, S.L.; Cleaves, H.J. Systems Biology: Geomics; Rigoutsos, I., Stephanopoulos, G., Eds.; Oxford Univessity Press: New York, NY, USA, 2007; Volume 1, pp. 3-56.

2. Bauer, H. Die ersten organisch-chemischen Synthesen. Naturwissenschaften 1980, 67, 1-6.

3. Proust, J.L. Contributions on Cyanides. Ann. Chim. Phys. 1806, 60, 233.

4. Wipperman, R. Ueber Tricyanwasserstoff, eine der blausaure polymere verbindung. Ber. Deustchen Chem. Ges. 1874, 7, 767-772.

5. Pflüger, E. Beitragë zur Lehre von der Respiration. I. Ueber die physiologische Verbrennung in den lebendigen organismen. Arch. Ges. Physiol. 1875, 10, 641-644.

6. Oró, J. Synthesis of adenine from ammonium cyanide. Biochem. Biophys. Res. Commun. 1960, 2, 407-412.

7. Gao, Y.; Solomon, P.M. HCN survey of normal spiral, infrared-luminous and ultraluminous galaxies. Astrophys. J. Suppl. Ser. 2004, 152, 63-80.

8. Buhl, D. Chemical constituents of interstellar clouds. Nature 1971, 234, 332-334.

9. Boger, G.I.; Sternberg, A. CN and HCN in dense interstellar clouds. Astrophys. J. 2005, 632, $302-315$.

10. Greaves, J.S.; Church, S.E. Photodissociation and the $\mathrm{CN}$ : HCN ratio: Observations of a 'Third Bar' in OMC1. Mon. Not. R. Astron. Soc. 1996, 283, 1179-1183.

11. Simon, R.; Stutzki, J.; Sternberg, A.; Winnewisser, G. Chemical stratification in the orion bar region: CN and CS submillimeter observations. Astron. Astrophys. 1997, 327, L9-L12.

12. Young Owl, R.C.; Meixner, M.M.; Wolfire, M.; Tielens, A.G.G.M.; Tauber, J. HCN and HCO ${ }^{+}$ images of the orion bar photodissociation region. Astrophys. J. 2000, 540, 886-906.

13. Savage, C.; Apponi, A.J.; Ziurys, L.M.; Wyckoff, S. Galactic ${ }^{12} \mathrm{C} /{ }^{13} \mathrm{C}$ Ratios from millimeter-wave observations of interstellar CN. Astrophys. J. 2002, 578, 211-223.

14. Schneider, N.; Simon, R.; Kramer, C.; Kraemer, K.; Stutzki, J.; Mookerjea, B. A multiwavelength study of the S 106 region-II. Characteristics of the photon dominated region. Astron. Astrophys. 2003, 406, 915-935.

15. Fuente, A.; Martin-Pintado, J.; Cernicharo, J.; Bachiller, R. A chemical study of the photodissociation region NGC 7023. Astron. Astrophys. 1993, 276, 473-488.

16. Fuente, A.; Martin-Pintado, J.; Gaume, R. High-density CN filaments in NGC 2023. Astrophys. J. 1995, 442, L33.

17. Jansen, D.J.; van Dishoeck, E.F.; Black, J.H.; Spaans, M.; Sosin, C. Physical and chemical structure of the IC 63 nebula. II. Chemical models. Astron. Astrophys. 1995, 302, 223-242. 
18. Fuente, A.; Rodriguez-Franc, A.; Garcia-Burillo, S.; Martin-Pintado, J.; Black, J.H. Observational study of reactive ions and radicals in PDRs. Astron. Astrophys. 2003, 406, 899-913.

19. Bachiller, R.; Forveille, T.; Huggins, P.J.; Cox, P. The chemical evolution of planetary nebulae. Astron. Astrophys. 1997, 324, 1123-1134.

20. McKay, C.P.; Borucki, W.J. Organic synthesis in experimental impact shocks. Science 1997, 276, 390-392.

21. Magee-Sauer, K.; Mumma, M.J.; DiSanti, M.A.; Russo, N.J.; Retting, T.W. Infrared spectroscopy of the $v_{3}$ band of hydrogen cyanide in Comet C/1995 O1 hale-bopp. Icarus 1999, 142, 498-508.

22. Wootten, A.; Lichten, S.M.; Sahai, R.; Wannier, P.G. CN abundance variations in the shell of IRC + 10216. Astrophys. J. 1982, 257, 151-160.

23. Truong-Bach, A.; Nguyen-Q-Rieu, A.; Omont, O.H.; Johansson, L.E.B. The circumstellar shell of IRC $+10216-$ Photo-chemistry of $\mathrm{C}_{2} \mathrm{H}$ and CN. Astron. Astrophys. 1987, 176, 285-293.

24. Bachiller, R.; Fuente, A.; Bujarrabal, V.; Colomar, F.; Loup, C.; Omont, A.; de Jong, T. A survey of CN in circumstellar envelopes. Astron. Astrophys. 1997, 319, 235-243.

25. Lindqvist, M.; Schçier, F.L.; Lucas, R.; Olofsson, H. Molecular envelopes around carbon stars Interferometric observations and models of $\mathrm{HCN}$ and $\mathrm{CN}$ emission. Astron. Astrophys. 2000, 361, 1036-1057.

26. Van Zadelhoff, G.-J.; Aikawa, Y.; Hogerheijde, M.R.; van Dishoeck, E.F. Axi-symmetric models of ultraviolet radiative transfer with applications to circumstellar disk chemistry. Astron. Astrophys. 2003, 397, 789-802.

27. Thi, W.-F.; van Zadelhoff, G.-J.; van Dishoeck, E.F. Organic molecules in protoplanetary disks around T Tauri and Herbig Ae stars. Astron. Astrophys. 2004, 425, 955-972.

28. Rank, M.D.; Townes, C.H.; Welch, W.J. Interstellar molecules and dense clouds. Science 1971, 174, 1083-1101.

29. Donn, B. Comets: Chemistry and chemical evolution. J. Mol. Evol. 1982, 18, 157-160.

30. Fray, N.; Bénilan, Y.; Cottin, H.; Gazeau, M.-C.; Crovisier, J. The origin of the CN radical in comets: A review from observations and models. Planet Space Sci. 2005, 53, 1243-1262.

31. Matthews, C.N. Hydrogen cyanide polymers from the impact of comet P/Shoemaker-Levy 9 on Jupiter. Adv. Space Res. 1997, 19, 1087-1091.

32. Pizzarello, S. Hydrogen cyanide in Murchinson meteorite. Astrophys. J. Lett. 2012, 754, L27.

33. Hanel, R.A.; Conrath, B.; Flaser, F.M.; Kunde, V.; Maguire, W.; Pearl, J.; Pirraglia, J.; Samuelson, R.; Herath, L.; Allison, M.; et al. Infrared observations of the saturnian system from voyager 1. Science 1981, 212, 192-200.

34. Tokunaga, A.T.; Beck, S.C.; Geballe, T.R.; Lacey, J.H.; Serabyn, E. The detection of HCN on Jupiter. Icarus 1981, 48, 283-289.

35. Owen, T. The atmosphere of Titan. J. Mol. Evol. 1982, 18, 150-156.

36. Irvine, W.M. The composition of interstellar molecular clouds. Space Sci. Rev. 1999, 90, 203-218.

37. Hidayat, T.; Marten, A.; Bézard, B.; Gautier, D.; Owen, T.; Matthwes, H.E.; Paubert, G. Millimeter and submillimeter heterodyne observations of Titan: Retrieval of the vertical profile of $\mathrm{HCN}$ and the ${ }^{12} \mathrm{C} /{ }^{13} \mathrm{C}$ ratio. Icarus $1997,126,170-182$. 
38. Hards, V. Volcanic contributions to the global carbon cycle. Sustainable and renewable energy. Br. Geol. Surv. Occas. Publ. 2005, 10, 16-17.

39. Ferris, J. Marine hydrothermal systems and the origin of life: Chemical markers of prebiotic chemistry in hydrothermal systems. Orig. Life Evol. Biosph. 1992, 22, 109-134.

40. Miller, S.L. The mechanism of synthesis of amino acids by electric discharges. Biochim. Biophys. Acta 1957, 23, 480-489.

41. Greenberg, J.M.; Mendoza-Gomez, C.X.; Pirronello, V. The Chemistry of life's origins. NATO ASI Ser. Ser. C Math. Phys. Sci. 1993, 416, 259-299.

42. Bar Nun, A.; Bar-Nun, N.; Bauer, S.H.; Sagan, C. Shock synthesis of amino acids in simulated primitive environments. Science 1970, 168, 470-473.

43. Ferris, J.P.; Chen, C.T. Photosynthesis of organic compounds in the atmosphere of Jupiter. Nature 1975, 258, 587-588.

44. Stribling, R.; Miller, S.L. Electric discharge synthesis of HCN in simulated Jovian Atmospheres. Orig. Life 1987, 17, 261.

45. Cataldo, F.; Lilla, E.; Ursini, O.; Angelini, G. TGA-FT-IR study of pirólisis of poly(hydrogen cyanide) synthesized from termal decomposition of formamide. Implications in cometary emissions. J. Anal. Appl. Pyrolysis 2010, 87, 34-44.

46. Ruiz-Bermejo, M.; de la Fuente, J.L.; Rogero, C.; Menor-Salván, C.; Osuna-Esteba, S.; Martín-Gago, J.A. New insights into the characterization of 'Insoluble Black HCN polymers'. Chem. Biodiver. 2012, 9, 25-40.

47. Mamajanov, I.; Herzfeld, J. HCN polymers characterized by SSNMR: Solid state reaction of crystalline tetramer (diaminomaleonitrile). J. Chem. Phys. 2009, 130, 134504.

48. Mamajanov, I.; Herzfeld, J. HCN polymers characterized by solid state NMR: Chains and sheets formed in the neat liquid. J. Chem. Phys. 2009, 130, 134503.

49. Umemoto, K.; Takahashi, M.; Yokota, K. Studies on structure of HCN oligomers. Orig. Life 1987, 17, 283-293.

50. Ferris, J.P.; Edelson, E.H.; Auyeung, J.M.; Joshi, P.C. Structural studies on HCN oligomers. J. Mol. Evol. 1981, 17, 69-77.

51. Matthews, C.N.; Moser, R.E. Peptide synthesis from hydrogen cyanide and water. Nature 1967, 215, 1230-1234.

52. Völker, T. Polymere blausäure. Angew. Chem. 1960, 72, 379-384.

53. Pernot, P.; Carrasco, N.; Thissen, R.; Schmitz-Afonso, I. Tholinomics-chemical analysis of nitrogen-rich polymers. Anal. Chem. 2010, 82, 1371-1380.

54. Vuitton, V.; Bonnet, J.Y.; Frisari, M.; Thissen, R.; Quirico, E.; Dutuit, O.; Schmitt, B.; Le Roy, L.; Fray, N.; Cottin, H.; et al. Very high resolution mass spectrometry of HCN polymers and tholins. Faraday Discuss. 2010, 147, 495-508.

55. Hanczyc, M.M. Metabolism and motility in prebiotic structures. Phylosophi. Trans. B 2011, 366, 2885-2895.

56. De la Fuente, J.L.; Ruiz-Bermejo, M.; Menor-Salván, C.; Osuna-Esteban, S. Thermal characterization of HCN polymers by TG-MS, TG, DTA and DSC methods. Polym. Degrad. Stab. 2011, 96, 943-948. 
57. He, C.; Lin, G.; Upton, K.T.; Imanaka, H.; Smith, M.A. Structural investigation of HCN polymer isotopomers by solution-state multidimensional NMR. J. Phys. Chem. A 2012, 116, 4751-4759.

58. Ferris, J.P.; Hagan, W.J. HCN and Chemicals evolution: The possible role of cyano compounds in prebiotic síntesis. Tetrahedron 1984, 40, 1093-1120.

59. Schwartz, A.W.; Voet, A.B. Recent progress in the prebiotic chemistry of HCN. Orig. Life 1984, 14, 91-98.

60. Saladino, R.; Crestini, C.; Costanzo, G.; DiMauro, E. Advances in the prebiotic synthesis of nucleic acids bases: Implications for the origin of Life. Curr. Org. Chem. 2004, 8, 1425-1443.

61. Matthews, C.N.; Minard, R.D. Hydrogen cyanide polymers, comets and the origin of life. Faraday Discuss. 2006, 133, 393-401.

62. Miller, S.L. A production of amino acids under possible primitive Earth conditions. Science 1953, 117, 528-529.

63. Oró, J.; Kamat, S.S. Amino-acids synthesis from hydrogen cyanide under possible pritive Earth conditions. Nature 1961, 190, 442-443.

64. Sanchez, R.A.; Ferris, J.P.; Orgel, L.E. Studies in prebiotic synthesis II, Synthesis of purine precursors anda mino acids from aqueous hydrogen cyanide. J. Mol. Biol. 1967, 30, 223-252.

65. Stribling, R.; Miller, S.L. Energy yields for hydrogen cyanide and formaldehyde syntheses: The $\mathrm{HCN}$ and amino acid concentrations in the primitive ocean. Orig. Life 1987, 17, 261-273.

66. Miyakawa, S.; Cleaves, H.J.; Miller, S.L. The cold origin of life: B. Implications based on pyrimidines and purines produced from frozen ammonium cyanide solutions. Orig. Life Evol. Biosph. 2002, 32, 209-218.

67. Levy, M.; Miller, S.L.; Brinton, K.; Bada, J.L. Prebiotic synthesis of adenine and amino acids under Europa-like conditions. Icarus 2000, 145, 609-123.

68. Garzón, L.; Garzón, M.L. Radioactivity as a significant energy source in prebiotic synthesis. Orig. Life Evol. Biosph. 2001, 31, 3-13.

69. Abelson, P.H. Chemical events on the primitive Earth. Proc. Natl. Acad. Sci. USA 1966, 55, $1365-1372$.

70. Draganic, Z.; Draganic, I. Evidence for amino acids in hydrolysates of compounds formed by ionizing radiations. Orig. Life 1977, 8, 371-376.

71. Draganic, Z.D.; Niketic, V.; Jovanovic, S.; Draganic, I.G. The radiolysis of aqueous ammonium cyanide: Compounds of interest to chemical evolution studies. J. Mol. Evol. 1980, 15, 239-260.

72. Labadie, M.; Jensen, R.; Neuzil, E. Recherches sur I'évolution pré-biologique III. Les acides azulmiques noirs formés à partir du cyanure d'ammonium. Biochim. Biophys. Acta 1968, 165, $525-533$.

73. Moser, R.E.; Claggett, A.R.; Matthews, C.N. Peptide formation from aminomalononitrile (HCN trimer). Tetrahedron Lett. 1968, 9, 1605-1068.

74. Moser, R.E.; Claggett, A.R.; Matthews, C.N. Peptide formation from diaminomaleonitrile (HCN tetramer). Tetrahedron Lett. 1968, 9, 1599-1603.

75. Yuasa, S.; Flory, D.; Basile, B.; Oró, J. On the abiotic formation of amino acids I. HCN as precursors of amino acids detected in extracts of lunar samples II. Formation of HCN and amino acids from simulated mixtures of gases released from lunar samples. J. Mol. Evol. 1984, 20, 52-58. 
76. Labadie, M.; Jensen, R.; Neuzil, E. Recherches sur l'eévolution pré-biologique. I. Composition en amino-acides des microsphérules obtenues a partir du cyanure d'ammonium. Bull. Soc. Chim. Biol. 1967, 49, 673-682.

77. Lowe, C.U.; Ress, A.; Markham, F.R.S. Synthesis of complex organic compounds from simple precursors: Formation of amino-acids, amino-acid polymer, fatty acids and purines from ammonium cyanide. Nature 1963, 19, 219-222.

78. Oró, J.; Kimball, P. Synthesis of purines under possible primitive Earth conditions. I. Adenine from hydrogen cyanide. Arch. Biochem. Biophys. 1962, 94, 217-227.

79. Ferris, J.P.; Wos, J.D.; Nooner, D.W.; Oró, J. Chemical evolution XXI. The amino acids released on hydrolysis of HCN oligomers. J. Mol. Evol. 1974, 3, 225-231.

80. Ferris, J.P.; Donner, D.B.; Lobo, A.P. Possible role of hydrogen cyanide in chemical evolution: Investigation of prposed direct synthesis of peptides from hydrogen cyanide. J. Mol. Biol. 1973, 74, 499-510.

81. Ferris, J.P.; Wos, J.D.; Ryan, T.J.; Lobo, A.P.; Donner, D.B. Biomolecules from HCN. Orig. Life 1974, 5, 153-157.

82. Ferris, J.P.; Joshi, P.C.; Edelson, E.H.; Lawless, J.G. HCN: A plausible source of purines, pyrimidines and amino acids on the primitive Earth. J. Mol. Evol. 1978, 11, 293-311.

83. Mizutani, H.; Mikuni, H.; Takahasi, M.; Noda, H. Study on the photochemical reaction of HCN and its polymers products relating to primary chemical evolution. Orig. Life 1975, 6, 513-525.

84. Sweeney, M.A.; Toste, A.P.; Ponnamperuma, C. Formation of amino acids by Cobalt-60 irradiation of hydrogen cyanide solutions. Orig. Life 1976, 7, 187-189.

85. Ferris, J.P.; Wos, J.D.; Lobo, A.P. Chemical Evolution. XXII. The hydantoins released on hydrolysis of HCN oligomers. J. Mol. Evol. 1974, 3, 311-316.

86. Ferris, J.P.; Donner, D.B.; Lobo, A.P. Possible role of hydrogen cyanide in Chemicals evolution: The oligomerization and condensation of hydrogen cyanide. J. Mol. Biol. 1973, 74, 511-518.

87. Miller, S.L.; Orgel, L.E. The Origins of Life on the Earth; Prentice-Hall: New York, NY, USA, 1974.

88. Gilbert, W. The origin of life: The RNA world. Nature 1986, 319, 618.

89. Sheppard, T.P.; Ordoukhanian, P.; Joyce, G.F. A DNA enzyme with N-glycosylase activity. Proc. Natl. Acad. Sci. USA 2000, 97, 7802-7807.

90. Santoro, S.W.; Joyce, G.F.; Sakthivel, K.; Gramatikova, S.; Barbas, C.F., III. RNA cleavage by a DNA enzymewith extended chemical functionality. J. Am. Chem. Soc. 2000, 122, 2433-2439.

91. Pullman, B. Electronic Factors in Biochemical Evolution. In Exobiology; Ponnamperuma, C., Ed.; North Holland Publishing Company: Amsterdam, The Netherlands and London, UK, 1972; p. 140.

92. Sanchez, R.A.; Ferris, J.P.; Orgel, L.E. Conditions for purine synthesis: Did prebiotic synthesis occur at low temperatures? Science 1966, 153, 72-73.

93. Schwartz, A.W.; Joosten, H.; Voet, A.B. Prebiotic adenine synthesis via HCN oligomerization in ice. BioSystems 1982, 15, 191-193.

94. Schwartz, A.W.; Goverde, M. Acceleration of HCN oligomerization by formaldehyde and related compounds: Implications for prebiotic synthesis. J. Mol. Evol. 1982, 18, 351-353.

95. Voet, A.B.; Schwartz, A.W. Prebiotic adenine synthesis from HCN-Evidence for a newly discorvered major pathway. Bioorg. Chem. 1983, 12, 8-17.

96. Schwartz, A.W.; Bakker, C.G. Was adenine the first purine? Science 1989, 245, 1102-1104. 
97. Negrón-Mendoza, A.; Draganic, Z.D. Search for heterocyclic radiolytic products in aqueous Solutions of cyanide. Adv. Space Res. 1984, 4, 121-124.

98. Borquez, E.; Cleaves, H.J.; Lazcano, A.; Miller, S.L. An investigation of prebiotic purine synthesis from the hydrolysis of HCN polymers. Orig. Life Evol. Biosph. 2005, 35, 79-90.

99. Levy, M.; Miller, S.L.; Oró, J. Production of guanine from $\mathrm{NH}_{4} \mathrm{CN}$ polymerizations. J. Mol. Evol. 1999, 49, 165-168.

100. Ferris, J.P.; Orgel, L.E. An inusual photochemical rearrangement in the shyntesis of adenine from hydrogen cyanide. J. Am. Chem. Soc. 1966, 88, 1074-1074.

101. Ferris, J.P.; Orgel, L.E. Studies in Prebiotic Synthesis. I. Aminomalononitrile and 4-Amino-5-cyanoimidazole. J. Am. Chem. Soc. 1966, 88, 3829-3831.

102. Ferris, J.P.; Joshi, P.C.; Lawless, J.G. Chemical evolution XXIX. Pyrimidines from hydrogen cyanide. BioSystems 1977, 9, 81-86.

103. Voet, A.B.; Schwartz, A.W. Uracil synthesis via HCN oligomerization. Orig. Life 1982, 12, 45-49.

104. Negrón-Mendoza, A.; Draganic, Z.D.; Navarro-Gonzalez, R.; Draganic, I.G. Aldehydes, ketones, and carboxylic acids formed radiolytically in aqueous Solutions of cyanides and simple nitriles. Rad. Res. 1983, 95, 248-261.

105. Negrón-Mendoza, A.; Ramos-Bernal, S.; Cruz, E.; Juárez, J.M. Radiolysis of HCN in heterogeneous phase. Rad. Phys. Chem. 2001, 61, 771-772.

106. Eschenmoser, A. On a hypothetical generational relationship between HCN and constituents of the reductive citric acid cycle. Chem. Biodiver. 2007, 4, 554-573.

107. Smith, E.; Morowitz, H.J. Universality in intermediary metabolism. Proc. Natl. Acad. Sci. USA 2004, 101, 13168-13173.

108. Eschenmoser, A.; Loewenthal, E. Chemistry of potentially prebiological natural products. Chem. Soc. Rev. 1992, 21, 1-16.

109. Eschenmosr, A. Vitamin $\mathrm{B}_{\mathrm{I} 2}$ : Experiments concerning the origin of its molecular structure. Angew. Chem. Int. Ed. 1988, 27, 5-39.

110. Visser, C.M. Evolutionary roots of catalysis by nicotinamida and flavins in C-H oxidoreductases and in photosynthesis. Orig. Life 1982, 12, 165-179.

111. Schimpl, A.; Lemmon, R.M.; Calvin, M. Cyanamide formation under primitive Earth conditions. Science 1965, 147, 149-150.

(C) 2013 by the authors; licensee MDPI, Basel, Switzerland. This article is an open access article distributed under the terms and conditions of the Creative Commons Attribution license (http://creativecommons.org/licenses/by/3.0/). 